\title{
THE LITTLEWOOD-GOWERS PROBLEM
}

\author{
T. SANDERS
}

\begin{abstract}
The paper has two main parts. To begin with suppose that $G$ is a compact Abelian group. Chang's Theorem can be viewed as a structural refinement of Bessel's inequality for functions $f \in L^{2}(G)$. We prove an analogous result for functions $f \in A(G)$, where $A(G)$ is the space $\left\{f \in L^{1}(G):\|\widehat{f}\|_{1}<\infty\right\}$ endowed with the norm $\|f\|_{A(G)}:=\|\widehat{f}\|_{1}$, and generalize this to the approximate Fourier transform on Bohr sets.

As an application of the first part of the paper we improve a recent result of Green and Konyagin: Suppose that $p$ is a prime number and $A \subset \mathbb{Z} / p \mathbb{Z}$ has density bounded away from 0 and 1 by an absolute constant. Green and Konyagin have shown that $\left\|\chi_{A}\right\|_{A(\mathbb{Z} / p \mathbb{Z})} \gg_{\varepsilon}(\log p)^{1 / 3-\varepsilon}$; we improve this to $\left\|\chi_{A}\right\|_{A(\mathbb{Z} / p \mathbb{Z})} \gg_{\varepsilon}(\log p)^{1 / 2-\varepsilon}$. To put this in context it is easy to see that if $A$ is an arithmetic progression then $\left\|\chi_{A}\right\|_{A(\mathbb{Z} / p \mathbb{Z})} \ll \log p$.
\end{abstract}

\section{INTRODUCTION}

We use the Fourier transform on compact Abelian groups, the basics of which may be found in Chapter 1 of Rudin [Rud90]; we take a moment to standardize our notation.

Suppose that $G$ is a compact Abelian group. Write $\widehat{G}$ for the dual group, that is the discrete Abelian group of continuous homomorphisms $\gamma: G \rightarrow S^{1}$, where $S^{1}:=\{z \in \mathbb{C}:|z|=1\}$. Although the natural group operation on $\widehat{G}$ corresponds to pointwise multiplication of characters we shall denote it by ' + ' in alignment with contemporary work. $G$ may be endowed with Haar measure $\mu_{G}$ normalised so that $\mu_{G}(G)=1$ and as a consequence we may define the Fourier transform . : $L^{1}(G) \rightarrow \ell^{\infty}(\widehat{G})$ which takes $f \in L^{1}(G)$ to

$$
\widehat{f}: \widehat{G} \rightarrow \mathbb{C} ; \gamma \mapsto \int_{x \in G} f(x) \overline{\gamma(x)} d \mu_{G}(x) .
$$

We write

$$
A(G):=\left\{f \in L^{1}(G):\|\widehat{f}\|_{1}<\infty\right\}
$$

and define a norm on $A(G)$ by $\|f\|_{A(G)}:=\|\widehat{f}\|_{1}$.

We are concerned with the following analogue of a question due to Littlewood, closely related to a problem of Gowers', which was first addressed by Green and Konyagin in GK09.

Question 1.1. Suppose that $p$ is a prime number and $A \subset \mathbb{Z} / p \mathbb{Z}$ has density bounded away from 0 and 1 by an absolute constant. How small can $\left\|\chi_{A}\right\|_{A(\mathbb{Z} / p \mathbb{Z})}$ be?

In their paper Green and Konyagin prove the following result. 
Theorem 1.2. Suppose that $p$ is a prime number and $A \subset \mathbb{Z} / p \mathbb{Z}$ has density bounded away from 0 and 1 by an absolute constant. Then

$$
\left\|\chi_{A}\right\|_{A(\mathbb{Z} / p \mathbb{Z})} \gg\left(\frac{\log p}{\log \log p}\right)^{1 / 3} .
$$

By analogy with the original problem of Littlewood they observe that more is probably true, indeed one might make the following conjecture.

Conjecture 1.3. (Green-Konyagin-Littlewood conjecture) Suppose that $p$ is a prime number and $A \subset \mathbb{Z} / p \mathbb{Z}$ has density bounded away from 0 and 1 by an absolute constant. Then

$$
\left\|\chi_{A}\right\|_{A(\mathbb{Z} / p \mathbb{Z})} \gg \log p .
$$

Certainly no more than this is true as any arithmetic progression of density bounded away from 0 and 1 shows.

In this paper we improve Theorem 1.2, increasing the exponent of $\log p$ from $1 / 3-\varepsilon$ to $1 / 2-\varepsilon$. Specifically we show the following.

Theorem 1.4. Suppose that $p$ is a prime number and $A \subset \mathbb{Z} / p \mathbb{Z}$ has density bounded away from 0 and 1 by an absolute constant. Then

$$
\left\|\chi_{A}\right\|_{A(\mathbb{Z} / p \mathbb{Z})} \gg\left(\frac{\log p}{(\log \log p)^{3}}\right)^{1 / 2} .
$$

The rest of the paper breaks into three parts as follows.

- $\S ₫ 2$.5. In these sections we develop a new structure theorem (Theorem 2.2) for the Fourier spectrum of functions in $A(G)$.

- $\$ ₫ 6$ [7 Here we recall the basic facts of local Fourier analysis and localize our new structure theorem in Proposition 7.4

- $\{\$ 8[11$ Finally we address the problem in this introduction and use the local version of our structure theorem to prove Theorem 1.4.

It is the results of the first two parts which seem most likely to have further uses; Theorem 1.4 should be seen as an application, albeit the motivating one, of these new tools.

\section{A structural Result for the Fourier spectrum}

One might begin studying the structure of the Fourier spectrum of functions by looking at the sets of characters at which $\widehat{f}$ is large; a natural realization of these sets is the sets

$$
\left\{\gamma \in \widehat{G}:|\widehat{f}(\gamma)| \geq \epsilon\|f\|_{1}\right\} \text { for } \epsilon \in(0,1] .
$$

The study of these sets has been surveyed by Green in Gre04 so we are brief and only recall the key facts. Write $\Gamma=\left\{\gamma \in \widehat{G}:|\widehat{f}(\gamma)| \geq \epsilon\|f\|_{1}\right\}$. Bessel's inequality yields

$$
|\Gamma| \epsilon^{2}\|f\|_{1}^{2} \leq\|\widehat{f}\|_{2}^{2} \leq\|f\|_{2}^{2} \Rightarrow|\Gamma| \leq \epsilon^{-2}\left(\|f\|_{2}\|f\|_{1}^{-1}\right)^{2} .
$$

Since $G$ is compact the norms nest and in particular $\|f\|_{2}\|f\|_{1}^{-1} \geq 1$. Now, there is a result of Chang from Cha02 which refines (2.1) if $\|f\|_{2}\|f\|_{1}^{-1}$ is much larger than 1 . We require some further notation to state this. If $\Lambda$ is a set of characters on $G$ and $m \in \mathbb{Z}^{\Lambda}$ has finite support then put

$$
m . \Lambda:=\sum_{\lambda \in \Lambda} m_{\lambda} \cdot \lambda \text { and }|m|:=\sum_{\lambda \in \Lambda}\left|m_{\lambda}\right|,
$$


where the second '?' in the first definition is the natural action of $\mathbb{Z}$ on $\widehat{G}$. Write $\langle\Lambda\rangle$ for the set of all finite \pm -sums of elements of $\Lambda$, that is

$$
\langle\Lambda\rangle:=\left\{m . \Lambda: m \in\{-1,0,1\}^{\Lambda} \text { and }|m|<\infty\right\} .
$$

Theorem 2.1. (Chang's Theorem) Suppose that $G$ is a compact Abelian group, $f \in L^{2}(G)$ and $\Gamma=\left\{\gamma \in \widehat{G}:|\widehat{f}(\gamma)| \geq \epsilon\|f\|_{1}\right\}$ for some $\epsilon \in(0,1]$. Then there is a set of characters $\Lambda$ such that $\Gamma \subset\langle\Lambda\rangle$ and

$$
|\Lambda| \ll \epsilon^{-2}\left(1+\log \|f\|_{2}\|f\|_{1}^{-1}\right) \text {. }
$$

We develop an analogue of Chang's Theorem with $A(G)$ in place of $L^{2}(G)$; here it turns out that the natural realization of the sets of characters at which $\widehat{f}$ is large is the sets

$$
\left\{\gamma \in \widehat{G}:|\widehat{f}(\gamma)| \geq \epsilon\|f\|_{\infty}\right\} \text { for } \epsilon \in(0,1] .
$$

There is an easy analogue of (2.1): Write $\Gamma=\left\{\gamma \in \widehat{G}:|\widehat{f}(\gamma)| \geq \epsilon\|f\|_{\infty}\right\}$. Then

$$
|\Gamma| \epsilon\|f\|_{\infty} \leq\|\widehat{f}\|_{1}=\|f\|_{A(G)} \Rightarrow|\Gamma| \leq \epsilon^{-1}\left(\|f\|_{A(G)}\|f\|_{\infty}^{-1}\right) .
$$

A trivial instance of Hausdorff's inequality tells us that $\|f\|_{A(G)}\|f\|_{\infty}^{-1} \geq 1$, and indeed the quantity $\|f\|_{A(G)}\|f\|_{\infty}^{-1}$ plays the same rôle in $A(G)$ as the quantity $\|f\|_{2}\|f\|_{1}^{-1}$ does in $L^{2}(G)$. To complete the square then, we shall prove the following.

Theorem 2.2. Suppose that $G$ is a compact Abelian group, $f \in A(G)$ and $\Gamma=$ $\left\{\gamma \in \widehat{G}:|\widehat{f}(\gamma)| \geq \epsilon\|f\|_{\infty}\right\}$ for some $\epsilon \in(0,1]$. Then there is a set of characters $\Lambda$ such that $\Gamma \subset\langle\Lambda\rangle$ and

$$
|\Lambda| \ll \epsilon^{-1}\left(1+\log \|f\|_{A(G)}\|f\|_{\infty}^{-1}\right) .
$$

\section{The proof of Theorem 2.2}

We say that a set of characters $\Lambda$ is dissociated if

$$
m \in\{-1,0,1\}^{\Lambda} \text { and } m . \Lambda=0_{\widehat{G}} \text { imply that } m \equiv 0 .
$$

We have the following simple lemma regarding dissociated sets.

Lemma 3.1. Suppose that $G$ is a compact Abelian group, $\Gamma$ is a set of characters on $G$ and $\Lambda$ is a maximal dissociated subset of $\Gamma$. Then $\Gamma \subset\langle\Lambda\rangle$.

To prove this one supposes, for a contradiction, that there is a $\gamma \in \Gamma \backslash\langle\Lambda\rangle$. If one adds this $\gamma$ to $\Lambda$ it is easy to see that the resulting set is strictly larger and dissociated.

In view of this lemma Theorem 2.2 follows from:

Proposition 3.2. Suppose that $G$ is a compact Abelian group, $f \in A(G)$, and $\Lambda$ a dissociated subset of $\left\{\gamma \in \widehat{G}:|\widehat{f}(\gamma)| \geq \epsilon\|f\|_{\infty}\right\}$ for some $\epsilon \in(0,1]$. Then

$$
|\Lambda| \ll \epsilon^{-1}\left(1+\log \|f\|_{A(G)}\|f\|_{\infty}^{-1}\right) .
$$

We prove this using a standard inner product type argument for which we require an auxiliary measure.

Proposition 3.3. (Auxiliary measure) Suppose that $G$ is a compact Abelian group, $\Lambda$ a finite dissociated set of characters on $G$ and $\omega \in \ell^{\infty}(\Lambda)$ has $\|\omega\|_{\infty} \leq 1$. Then for any $\eta \in(0,1]$ there is a measure $\mu_{\eta} \in M(G)$ such that

$$
\left.\widehat{\mu_{\eta}}\right|_{\Lambda}=\omega,\left\|\mu_{\eta}\right\| \ll\left(1+\log \eta^{-1}\right) \text { and }\left|\widehat{\mu_{\eta}}(\gamma)\right| \leq \eta \text { for all } \gamma \notin \Lambda \text {. }
$$


Proof of Proposition 3.2. We define

$$
\omega(\lambda):=\frac{\widehat{f}(\lambda)}{|\widehat{f}(\lambda)|} \text { for all } \lambda \in \Lambda .
$$

$\omega \in \ell^{\infty}(\Lambda)$ and $\|\omega\|_{\infty} \leq 1$ so we may apply Proposition 3.3 to get the auxiliary measure $\mu_{\eta}$ corresponding to $\omega$. We examine the inner product $\left\langle f, \mu_{\eta}\right\rangle$.

$$
\begin{aligned}
\left|\left\langle f, \mu_{\eta}\right\rangle\right| & =\left|\sum_{\gamma \in \widehat{G}} \widehat{f}(\gamma) \overline{\widehat{\mu_{\eta}}(\gamma)}\right| \text { by Plancherel's theorem, } \\
& =\left|\sum_{\lambda \in \Lambda} \widehat{f}(\lambda) \overline{\widehat{\mu_{\eta}}(\lambda)}+\sum_{\gamma \notin \Lambda} \widehat{f}(\gamma) \overline{\widehat{\mu_{\eta}}(\gamma)}\right| \\
& \geq\left|\sum_{\lambda \in \Lambda} \widehat{f}(\lambda) \overline{\widehat{\mu_{\eta}}(\lambda)}\right|-\left|\sum_{\gamma \notin \Lambda} \widehat{f}(\gamma) \overline{\widehat{\mu_{\eta}}(\gamma)}\right| \\
& \geq\left|\sum_{\lambda \in \Lambda} \widehat{f}(\lambda) \overline{\omega(\lambda)}\right|-\eta \sum_{\gamma \notin \Lambda}|\widehat{f}(\gamma)| \text { from the properties of } \mu_{\eta}, \\
& \geq \sum_{\lambda \in \Lambda}|\widehat{f}(\lambda)|-\eta\|f\|_{A(G)} \\
& \geq|\Lambda| \epsilon\|f\|_{\infty}-\eta\|f\|_{A(G)} .
\end{aligned}
$$

However

$$
\left|\left\langle f, \mu_{\eta}\right\rangle\right| \leq\|f\|_{\infty}\left\|\mu_{\eta}\right\| \ll\|f\|_{\infty}\left(1+\log \eta^{-1}\right),
$$

so that

$$
\|f\|_{\infty}\left(1+\log \eta^{-1}\right) \gg|\Lambda| \epsilon\|f\|_{\infty}-\eta\|f\|_{A(G)} .
$$

Choosing $\eta^{-1}=\|f\|_{A(G)}\|f\|_{\infty}^{-1}$ yields the result.

\section{Constructing the auxiliary measure}

The construction of the auxiliary measure is best illustrated in the model setting of $\mathbb{F}_{2}^{n}$ where we benefit from two simplifications. Suppose that $\Lambda$ is a set of characters on $\mathbb{F}_{2}^{n}$. Then

- $\langle\Lambda\rangle$ is simply the subspace of $\widehat{G}$ generated by $\Lambda$;

- $\Lambda$ is dissociated if and only if it is linearly independent over $\widehat{\mathbb{F}_{2}^{n}}$.

The first of these is simply a convenience while the second represents the major obstacle in transferring the arguments of this section to the general setting. We shall prove the following result.

Proposition 4.1. Suppose that $\Lambda$ is a linearly independent set of characters on $\mathbb{F}_{2}^{n}$ and $\omega: \Lambda \rightarrow[-1,1]$. Then for any $\eta \in(0,1]$ there is a measure $\mu_{\eta} \in M\left(\mathbb{F}_{2}^{n}\right)$ such that

$$
\left.\widehat{\mu_{\eta}}\right|_{\Lambda}=\omega,\left\|\mu_{\eta}\right\| \ll\left(1+\log \eta^{-1}\right) \text { and }\left|\widehat{\mu_{\eta}}(\gamma)\right| \leq \eta \text { for all } \gamma \notin \Lambda .
$$

We relegate the technical process of extending this construction to arbitrary compact Abelian groups to Appendix $\mathrm{A}$.

Riesz products are the building blocks of the measure, the necessary details of which we now record. 
4.2. Riesz products. Suppose that $\Lambda$ is a finite set of characters. If $\omega: \Lambda \rightarrow$ $[-1,1]$ then we define the product

$$
p_{\omega}:=\prod_{\lambda \in \Lambda}(1+\omega(\lambda) \lambda) .
$$

Such a product is called a Riesz product and it is easy to see that it is real and non-negative from which it follows that $\left\|p_{\omega}\right\|_{1}=\widehat{p_{\omega}}\left(0_{\widehat{G}}\right)$. Further, expanding out the product reveals that, $\operatorname{supp} \widehat{p_{\omega}} \subset\langle\Lambda\rangle$.

If $\Lambda$ is linearly independent then we can easily compute the Fourier transform of a Riesz product. Suppose that $\gamma \in\langle\Lambda\rangle$, then there is a unique $m: \Lambda \rightarrow\{0,1\}$ such that $\gamma=m . \Lambda$ by the linear independence of $\Lambda$, so

$$
\widehat{p_{\omega}}(\gamma):=\prod_{\substack{\lambda \in \Lambda \\ m_{\lambda} \neq 0}} \omega(\lambda) .
$$

This leads to the observation that $\left\|p_{\omega}\right\|_{1}=\widehat{p_{\omega}}\left(0_{\widehat{G}}\right)=1$ and $\left.\widehat{p_{\omega}}\right|_{\Lambda}=\omega$. Moreover if $t \in[-1,1]$ then

$$
\widehat{p_{t \omega}}(m . \Lambda):=t^{|m|} \widehat{p_{\omega}}(m . \Lambda) \text { where, as before, }|m|=\sum_{\lambda \in \Lambda}\left|m_{\lambda}\right|,
$$

So, if $|m|>1$ then

$$
\left|\widehat{p_{t \omega}}(m . \Lambda)\right| \leq|t|^{2}\left|\widehat{p_{\omega}}(m . \Lambda)\right| \leq|t|^{2}\left\|p_{\omega}\right\|_{1}=|t|^{2}
$$

It follows that a lot of the Fourier coefficients of $p_{t \omega}$ are already small if $|t|$ is small. By taking $\mu_{\eta}:=\eta^{-1} p_{\eta \omega}$ we get a well known primitive version of the auxiliary measure of Proposition 3.3.

Proposition 4.3. (Primitive auxiliary measure) Suppose that $\Lambda$ is a linearly independent set of characters on $\mathbb{F}_{2}^{n}$ and $\omega: \Lambda \rightarrow[-1,1]$. Then for any $\eta \in(0,1]$ there is a measure $\mu_{\eta} \in M\left(\mathbb{F}_{2}^{n}\right)$ such that

$$
\left.\widehat{\mu_{\eta}}\right|_{\Lambda}=\omega,\left\|\mu_{\eta}\right\| \ll \eta^{-1} \text { and }\left|\widehat{\mu_{\eta}}(\gamma)\right| \leq \eta \text { for all } \gamma \notin \Lambda \cup\left\{0_{\widehat{G}}\right\} \text {. }
$$

The basic idea for improving the measure of Proposition 4.3 rests on the observation that if $|m|$ is large then $\left|\widehat{p_{t \omega}}(m . \Lambda)\right|$ is in fact guaranteed to be very small. To construct a better measure we take linear combinations of Riesz products so that their Fourier transforms cancel on the characters $m . \Lambda$ where $|m|$ is small (except of course for $|m|=1)$. Begin by considering

$$
\nu_{t}:=\frac{1}{2}\left(p_{t \omega}-p_{-t \omega}\right) .
$$

Then

$$
\left.\widehat{\nu_{t}}\right|_{\Lambda}=t \omega,\left\|\nu_{t}\right\| \leq 1,\left|\widehat{\nu_{t}}(m . \Lambda)\right| \leq t^{|m|}
$$

and

It follows that

$$
\widehat{\nu_{t}}(m \cdot \Lambda)=0 \text { if }|m| \equiv 0 \quad(\bmod 2) .
$$

$$
\left.\widehat{\nu_{t}}\right|_{\Lambda}=t \omega,\left\|\nu_{t}\right\| \leq 1 \text { and }\left|\widehat{\nu_{t}}(\gamma)\right| \leq t^{3} \text { for all } \gamma \notin \Lambda .
$$

If we put $\mu_{\eta}=\nu_{\sqrt{\eta}}$ then we have a refinement of Proposition 4.3 with $\left\|\mu_{\eta}\right\| \ll \eta^{-1 / 2}$ instead of $\left\|\mu_{\eta}\right\| \ll \eta^{-1}$. 
More generally we consider a measure $\tau$ on $[-1,1]$ and put

$$
\nu_{\tau}:=\int p_{t \omega} d \tau(t)
$$

Then

$$
\left\|\nu_{\tau}\right\| \leq \sup _{t \in[-1,1]}\left\|p_{t \omega}\right\| \cdot\|\tau\|=\|\tau\| \text { and } \widehat{\nu_{\tau}}(m . \Lambda)=\int t^{|m|} d \tau(t) \widehat{p_{\omega}}(m . \Lambda) .
$$

Following the idea of trying to get the Fourier transforms of the Riesz products in $\nu_{\tau}$ to cancel on $\{m . \Lambda:|m|=r\}$, we should like a measure $\tau_{l}$ with $\left\|\tau_{l}\right\|$ minimal subject to

$$
\int t^{k} d \tau_{l}(t)=0 \text { for } 1<k \leq l, \int d \tau_{l}(t)=0, \text { and } \int t d \tau_{l}(t)=1 .
$$

Méla, in Mél82, already had this idea, and moreover for the purpose of constructing essentially the auxiliary measure we want. To produce $\tau_{l}$ he constructs a measure $\sigma_{l}$ with the following properties:

Lemma 4.4. (Lemma 4, $\S 7$, Mél82) Suppose that $l>1$ is an integer. Then there is a measure $\sigma_{l}$ on $[0,1]$ such that

$$
\int s^{2 k-1} d \sigma_{l}(s)=0 \text { for } 2 \leq k \leq l, \int s d \sigma_{l}(s)=1 \text { and }\left\|\sigma_{l}\right\|=2 l-1 .
$$

He chooses $\sigma_{l}$ to be (the measure induced by) the polynomial $s+\left((-1)^{l} /(2 l-\right.$ 1)) $P_{2 l-1}(s)$ where $P_{2 l-1}$ is the Chebychev polynomial of order $2 l-1$. Once this is known it is not hard to verify the properties of $\sigma_{l}$.

We take $\tau_{2 l}$ to be the odd measure on $[-1,1]$ which extends $2 \sigma_{l}(2 s)$ on $[0,1 / 2]$, and the null measure on $[1 / 2,1]$. It is easy, then, to verify the following.

Lemma 4.5. Suppose that $l>1$ is an integer. Then the measure $\tau_{2 l}$ on $[-1,1]$ has $\left\|\tau_{2 l}\right\| \leq 2(2 l-1)$,

$$
\int t^{k} d \tau_{2 l}(t)=\left\{\begin{array}{l}
0 \text { if } k \leq 2 l \text { and } k \neq 1 \\
1 \text { if } k=1
\end{array}\right.
$$

and $\left|\int t^{k} d \tau_{2 l}(t)\right| \leq 2^{1-k}$ for all $k$.

Proposition 4.1 follows from this by taking $\mu_{\eta}=\nu_{\tau_{2 l}}$ with $l=\left\lceil 2^{-1} \log _{2} \eta^{-1}\right\rceil$.

\section{Remarks on Theorem 2.2}

The technique of applying Lemma3.1 to reduce Theorem2.2 to Proposition3.2 is used by Chang, Cha02, in the proof of Theorem 2.1. The analogue of Proposition 3.2 in that case is proved using the dual formulation of Rudin's inequality, which states that if $\Lambda$ is a dissociated set of characters on $G$ and $f \in L^{2}(G)$ then

$$
\left\|\left.\widehat{f}\right|_{\Lambda}\right\|_{2} \ll \sqrt{\frac{p}{p-1}}\|f\|_{p} \text { for } 2 \geq p>1 .
$$

Halász, Hal81, uses the inner product technique of Proposition 3.2 to prove a nonFourier result in discrepancy theory and employs a Riesz product (for a different Hilbert space) as the auxiliary measure. An exposition of his result may be found in Chazelle [Cha00] and this was the original motivation for our result.

Green pointed out the fact that Méla, in Mél82, had already used the method of linear combinations of Riesz products to construct the auxiliary measure we require. 
Méla uses it as an example to show that a result of his regarding $\epsilon$-idempotent measures is essentially best possible. In fact it follows from Méla's result that essentially no better auxiliary measure than the one we have constructed exists.

Finally, Proposition 3.2, like its analogue for Chang's theorem, can be proved for a wider class of sets than simply dissociated sets, namely Sidon sets. If $G$ is a compact Abelian group and $\Lambda$ a set of characters on $G$ then $\Lambda$ is a Sidon set if every $\phi \in \ell^{\infty}(\Lambda)$ has the form $\left.\widehat{\mu}\right|_{\Lambda}$ for some $\mu \in M(G)$. Extending the results to Sidon sets comes down to constructing auxiliary measures for this class of sets. Drury, in Dru72, proved Proposition 4.3 for Sidon sets of characters on general compact Abelian groups, and his methods can be used to extend Proposition 3.3 .

\section{Local Fourier analysis in COMPaCt Abelian groups}

Our attention now turns to developing the tools of local Fourier analysis and localizing the results of the previous four sections.

Given $f \in L^{1}(G)$ we often want to approximate $f$ by a less complicated function. One way to do this is to approximate $f$ by its expectation on level sets of characters. To analyze the error in so doing we restrict the function to these level sets and use the Fourier transform on the restricted function.

If $\Gamma$ is a finite set of characters then we define the annihilator of $\Gamma$ to be

$$
\Gamma^{\perp}:=\{x \in G: \gamma(x)=1 \text { for all } \gamma \in \Gamma\} .
$$

If $x^{\prime}+\Gamma^{\perp}$ (a maximal joint level set of the characters in $\Gamma$ ) has positive measure in $G$ then it is easy to localize the Fourier transform to $x^{\prime}+\Gamma^{\perp}$ :

$$
L^{1}\left(x^{\prime}+\Gamma^{\perp}\right) \rightarrow \ell^{\infty}(\widehat{G}) ; f \mapsto f d\left(\widehat{x^{\prime}+\mu_{\Gamma^{\perp}}}\right) .
$$

Note that the right hand side is constant on cosets of $\Gamma^{\perp \perp}$ (defined in the obvious manner) and so can be thought of as an element of $\ell^{\infty}\left(\widehat{G} / \Gamma^{\perp \perp}\right)$.

Bourgain, in Bou99, observed that one can localize the Fourier transform to typical approximate level sets and retain approximate versions of a number of the standard results for the Fourier transform on compact Abelian groups. Since his original work various expositions and extensions have appeared most notably in the various papers of Green and Tao. Indeed all the results of this section can be found in GT08, for example.

6.1. Approximate annihilators: Bohr neighborhoods and some of their properties. Throughout this section $G$ is a compact Abelian group, $\Gamma$ a non-empty finite set of characters on $G$ and $\delta \in(0,1]$.

We can define a natural valuation on $S^{1}$, namely

$$
\|z\|:=\frac{1}{2 \pi} \inf _{n \in \mathbb{Z}}|2 \pi n+\arg z|,
$$

which can be used to measure how far $\gamma(x)$ is from 1 . Consequently we define a prototype for an approximate annihilator:

$$
B(\Gamma, \delta):=\{x \in G:\|\gamma(x)\| \leq \delta \text { for all } \gamma \in \Gamma\},
$$

called a Bohr set. A translate of such a set is called a Bohr neighborhood. We adopt the convention that if $B(\Gamma, \delta)$ is a Bohr set then the size of $\Gamma$ is denoted by $d$.

Bohr sets are easily seen to be closed. To ensure that they have positive measure we recall the following easy application of the pigeonhole principle. 
Lemma 6.2. Suppose $G$ is a compact Abelian group and $B(\Gamma, \delta)$ is a Bohr set. Then $\mu_{G}(B(\Gamma, \delta)) \geq \delta^{d}$, where as our convention states, $d:=|\Gamma|$.

Hence we write $\beta_{\Gamma, \delta}$, or simply $\beta$ or $\beta_{\delta}$ if the parameters are implicit, for the measure induced on $B(\Gamma, \delta)$ by $\mu_{G}$, normalised so that $\left\|\beta_{\Gamma, \delta}\right\|_{1}=1$. This is sometimes referred to as the normalised Bohr cutoff. We write $\beta^{\prime}$ for $\beta_{\Gamma^{\prime}, \delta^{\prime}}$, or $\beta_{\Gamma, \delta^{\prime}}$ if no $\Gamma^{\prime}$ has been defined.

Annihilators are subgroups of $G$, a property which, at least in an approximate form, we would like to recover. Suppose that $\eta \in(0,1]$. Then $B(\Gamma, \delta)+B(\Gamma, \eta \delta) \subset$ $B(\Gamma,(1+\eta) \delta)$. If $B(\Gamma,(1+\eta) \delta)$ is not much bigger than $B(\Gamma, \delta)$ then we have a sort of approximate additive closure in the sense that $B(\Gamma, \delta)+B(\Gamma, \eta \delta) \approx B(\Gamma,(1+\eta) \delta)$. Not all Bohr sets have this property, however, Bourgain showed that typically they do. For our purposes we have the following proposition.

Proposition 6.3. Suppose that $G$ is a compact Abelian group, $\Gamma$ a set of $d$ characters on $G$ and $\delta \in(0,1]$. There is an absolute constant $c_{\mathcal{R}}>0$ and $a \delta^{\prime} \in[\delta / 2, \delta)$ such that

$$
\frac{\mu_{G}\left(B\left(\Gamma,(1+\kappa) \delta^{\prime}\right)\right)}{\mu_{G}\left(B\left(\Gamma, \delta^{\prime}\right)\right)}=1+O(|\kappa| d)
$$

whenever $|\kappa| d \leq c_{\mathcal{R}}$.

This result is not as easy as the rest of the section, it uses a covering argument; a nice proof can be found in GT08. We say that $\delta^{\prime}$ is regular for $\Gamma$ or that $B\left(\Gamma, \delta^{\prime}\right)$ is regular if

$$
\frac{\mu_{G}\left(B\left(\Gamma,(1+\kappa) \delta^{\prime}\right)\right)}{\mu_{G}\left(B\left(\Gamma, \delta^{\prime}\right)\right)}=1+O(|\kappa| d) \text { whenever }|\kappa| d \leq c_{\mathcal{R}} .
$$

It is regular Bohr sets to which we localize the Fourier transform and we begin by observing that regular Bohr cutoffs are approximately translation invariant and so function as normalised approximate Haar measures.

Lemma 6.4. (Normalized approximate Haar measure) Suppose that $G$ is a compact Abelian group and $B(\Gamma, \delta)$ is a regular Bohr set. If $y \in B\left(\Gamma, \delta^{\prime}\right)$ then $\|\left(y+\beta_{\delta}\right)-$ $\beta_{\delta} \| \ll d \delta^{\prime} \delta^{-1}$ where we recall that $y+\beta_{\delta}$ denotes the measure $\beta_{\delta}$ composed with translation by $y$.

Proof. Note that $\operatorname{supp}\left(\left(y+\beta_{\delta}\right)-\beta_{\delta}\right) \subset B\left(\Gamma, \delta+\delta^{\prime}\right) \backslash B\left(\Gamma, \delta-\delta^{\prime}\right)$ whence

$$
\left\|\left(y+\beta_{\delta}\right)-\beta_{\delta}\right\| \leq \frac{\mu_{G}\left(B\left(\Gamma, \delta+\delta^{\prime}\right) \backslash B\left(\Gamma, \delta-\delta^{\prime}\right)\right)}{\mu_{G}(B(\Gamma, \delta))} \ll d \delta^{\prime} \delta^{-1},
$$

by regularity.

In applications the following two simple corollaries will be useful but they should be ignored until they are used.

Corollary 6.5. Suppose that $G$ is a compact Abelian group and $B(\Gamma, \delta)$ is a regular Bohr set. If $\mu \in M\left(B\left(\Gamma, \delta^{\prime}\right)\right)$ then $\left\|\beta * \mu-\beta \int d \mu\right\| \ll\|\mu\| d \delta^{\prime} \delta^{-1}$.

Proof. The measures $\beta * \mu$ and $\beta \int d \mu$ agree inside $B\left(\Gamma, \delta-\delta^{\prime}\right)$ and outside $B(\Gamma, \delta+$ $\left.\delta^{\prime}\right)$, furthermore $\|\beta * \mu\| \leq\|\mu\|$ and $\left\|\beta \int d \mu\right\| \leq\|\mu\|$, whence

$$
\left\|\beta * \mu-\beta \int d \mu\right\| \ll\|\mu\| \frac{\mu_{G}\left(B\left(\Gamma, \delta+\delta^{\prime}\right) \backslash B\left(\Gamma, \delta-\delta^{\prime}\right)\right)}{\mu_{G}(B(\Gamma, \delta))} \ll\|\mu\| d \delta^{\prime} \delta^{-1}
$$

by regularity. 
Corollary 6.6. Suppose that $G$ is a compact Abelian group and $B(\Gamma, \delta)$ is a regular Bohr set. If $f \in L^{\infty}(G)$ then

$$
\|f * \beta-f * \beta(x)\|_{L^{\infty}\left(x+B\left(\Gamma, \delta^{\prime}\right)\right)} \ll\|f\|_{L^{\infty}(G)} d \delta^{\prime} \delta^{-1} .
$$

Proof. Note that

$$
\begin{aligned}
|f * \beta(x+y)-f * \beta(x)| & =|f *((-y+\beta)-\beta)(x)| \\
& \leq\|f\|_{L^{\infty}\left(\mu_{G}\right)}\|(-y+\beta)-\beta\| .
\end{aligned}
$$

The result follows by Lemma 6.4

With an approximate Haar measure we are in a position to define the local Fourier transform: Suppose that $x^{\prime}+B(\Gamma, \delta)$ is a regular Bohr neighborhood. Then we define the Fourier transform local to $x^{\prime}+B(\Gamma, \delta)$ by

$$
L^{1}\left(x^{\prime}+B(\Gamma, \delta)\right) \rightarrow \ell^{\infty}(\widehat{G}) ; f \mapsto f d\left({\widehat{x^{\prime}+\beta_{\Gamma, \delta}}}\right),
$$

where $L^{p}\left(x^{\prime}+B(\Gamma, \delta)\right)$ denotes the space

$$
\left\{f \in L^{1}(G): \operatorname{supp} f \subset x^{\prime}+B(\Gamma, \delta) \text { and } \int|f|^{p} d\left(x^{\prime}+\beta\right)<\infty\right\},
$$

equipped with the norm

$$
\|f\|_{L^{p}\left(x^{\prime}+B(\Gamma, \delta)\right)}:=\left(\int|f|^{p} d\left(x^{\prime}+\beta\right)\right)^{1 / p} .
$$

It is useful at this stage to also define $A\left(x^{\prime}+B(\Gamma, \delta)\right)$ which is the space

$$
\left\{f \in L^{1}(G): \operatorname{supp} f \subset x^{\prime}+B(\Gamma, \delta) \text { and }\|\widehat{f}\|_{1}<\infty\right\},
$$

equipped with the norm

$$
\|f\|_{A\left(x^{\prime}+B(\Gamma, \delta)\right)}=\|\widehat{f}\|_{1} .
$$

The translation of the Bohr set by $x^{\prime}$ simply twists the Fourier transform and is unimportant for the most part so we tend to restrict ourselves to the case when $x^{\prime}=0$.

$\widehat{f d \mu_{\Gamma^{\perp}}}$ was constant on cosets of $\Gamma^{\perp \perp}$. In the approximate setting have an approximate analogue on which $\widehat{g d \beta}$ does not vary too much. There are a number of possibilities:

$$
\begin{array}{cc}
\{\gamma:|1-\gamma(x)| \leq \epsilon \text { for all } x \in B(\Gamma, \delta)\} & \text { for } \epsilon \in(0,1] \\
\{\gamma:|1-\widehat{\beta}(\gamma)| \leq \epsilon\} & \text { for } \epsilon \in(0,1] \\
\{\gamma:|\widehat{\beta}(\gamma)| \geq \epsilon\} & \text { for } \epsilon \in(0,1]
\end{array}
$$

In applications each of these classes of sets is useful and so we should like all of them to be approximately equivalent. There is a clear chain of inclusions between the classes:

$\{\gamma:|1-\gamma(x)| \leq \epsilon$ for all $x \in B(\Gamma, \delta)\} \subset\{\gamma:|1-\widehat{\beta}(\gamma)| \leq \epsilon\} \subset\{\gamma:|\widehat{\beta}(\gamma)| \geq 1-\epsilon\}$ for $\epsilon \in(0,1]$. For a small cost in the width of the Bohr set we can ensure that the sets in the third class are contained in those in the first.

Lemma 6.7. Suppose that $G$ is a compact Abelian group and $B(\Gamma, \delta)$ is a regular Bohr set. Suppose that $\eta_{1}, \eta_{2}>0$. Then there is a $\delta^{\prime} \gg \eta_{1} \eta_{2} \delta / d$ such that

$$
\left\{\gamma:|\widehat{\beta}(\gamma)| \geq \eta_{1}\right\} \subset\left\{\gamma:|1-\gamma(x)| \leq \eta_{2} \text { for all } x \in B\left(\Gamma, \delta^{\prime}\right)\right\} \text {. }
$$


Proof. If $x \in B\left(\Gamma, \delta^{\prime}\right)$ then we have

$$
\eta_{1}|1-\gamma(x)| \leq|\widehat{\beta}(\gamma)||1-\overline{\gamma(x)}|=\mid\left((x \widehat{+\beta)}-\beta)(\gamma) \mid \ll d \delta^{\prime} \delta^{-1}\right.
$$

by Lemma 6.4. It follows that we may pick $\delta^{\prime} \gg \eta_{1} \eta_{2} \delta / d$ such that $|1-\gamma(x)| \leq \eta_{2}$ for all $x \in B\left(\Gamma, \delta^{\prime}\right)$.

This concludes the basic definitions of the local Fourier transform; in the next section we transfer the results we require to the local setting.

\section{A structural Result for the local Fourier spectrum}

In $\oint 2$ we recorded a number of results regarding the structure of the collection of characters supporting large values of the Fourier transform; in this section we examine local versions of these. About the simplest statement we made was (2.1) which asserted that if $f \in L^{2}(G)$ and $\Gamma=\left\{\gamma:|\widehat{f}(\gamma)| \geq \epsilon\|f\|_{1}\right\}$ then

$$
|\Gamma| \leq \epsilon^{-2}\left(\|f\|_{2}\|f\|_{1}^{-1}\right)^{2} .
$$

The following analogue for functions $f \in L^{2}(B(\Gamma, \delta))$ was proved by Green and Tao in GT08, by localizing Bessel's inequality.

Proposition 7.1. Suppose that $G$ is a compact Abelian group and $B(\Gamma, \delta)$ a regular Bohr set. Suppose that $f \in L^{2}(B(\Gamma, \delta))$ and $\epsilon, \eta \in(0,1]$. Write $L_{f}$ for the quantity $\|f\|_{L^{2}(B(\Gamma, \delta))}\|f\|_{L^{1}(B(\Gamma, \delta))}^{-1}$. Then there is a set $\Lambda$ of characters and a $\delta^{\prime} \in(0,1]$ with

$$
|\Lambda| \ll \epsilon^{-2} L_{f}^{2} \text { and } \delta^{\prime} \gg \epsilon^{2} \eta \delta / d L_{f}^{2}
$$

such that

$\left\{\gamma \in \widehat{G}:|\widehat{f d \beta}(\gamma)| \geq \epsilon\|f\|_{L^{1}(B(\Gamma, \delta))}\right\} \subset\left\{\gamma:|1-\gamma(x)| \leq \eta\right.$ for all $\left.x \in B\left(\Gamma \cup \Lambda, \delta^{\prime}\right)\right\}$.

Later on in $\$ 2$ we noted (2.2), an analogue of (2.1) for functions $f \in A(G)$, and there is a corresponding local analogue of this result for $f \in A(B(\Gamma, \delta))$ which we note now.

Proposition 7.2. Suppose that $G$ is a compact Abelian group and $B(\Gamma, \delta)$ a regular Bohr set. Suppose that $f \in A(B(\Gamma, \delta))$ and $\epsilon, \eta \in(0,1]$. Write $A_{f}$ for the quantity $\|f\|_{A(B(\Gamma, \delta))}\|f\|_{L^{\infty}(B(\Gamma, \delta))}^{-1}$. Then there is a set $\Lambda$ of characters and a $\delta^{\prime} \in(0,1]$ with

$$
|\Lambda| \ll \epsilon^{-1} A_{f} \text { and } \delta^{\prime} \gg \epsilon \eta \delta / d A_{f}
$$

such that

$\left\{\gamma \in \widehat{G}:|\widehat{f d \beta}(\gamma)| \geq \epsilon\|f\|_{L^{\infty}(B(\Gamma, \delta))}\right\} \subset\left\{\gamma:|1-\gamma(x)| \leq \eta\right.$ for all $\left.x \in B\left(\Gamma \cup \Lambda, \delta^{\prime}\right)\right\}$.

In San08 the result of Green and Tao is refined with Chang's theorem. One can use the same techniques to refine Proposition 7.2 with Theorem 2.2, our $A(G)$ analogue of Chang's theorem; doing so gives the following.

Proposition 7.3. Suppose that $G$ is a compact Abelian group and $B(\Gamma, \delta)$ a regular Bohr set. Suppose that $f \in A(B(\Gamma, \delta))$ and $\epsilon, \eta \in(0,1]$. Write $A_{f}$ for the quantity $\|f\|_{A(B(\Gamma, \delta))}\|f\|_{L^{\infty}(B(\Gamma, \delta))}^{-1}$. Then there is a set $\Lambda$ of characters and a $\delta^{\prime} \in(0,1]$ with

$$
|\Lambda| \ll \epsilon^{-1}\left(1+\log A_{f}\right) \text { and } \delta^{\prime} \gg \epsilon \eta \delta / d^{2}\left(1+\log A_{f}\right),
$$

such that

$\left\{\gamma \in \widehat{G}:|\widehat{f d \beta}(\gamma)| \geq \epsilon\|f\|_{L^{\infty}(B(\Gamma, \delta))}\right\} \subset\left\{\gamma:|1-\gamma(x)| \leq \eta\right.$ for all $\left.x \in B\left(\Gamma \cup \Lambda, \delta^{\prime}\right)\right\}$. 
For our application we are in fact able to assume that $f \in A(G)$, and in that case we have the following slightly more general conclusion.

Proposition 7.4. Suppose that $G$ is a compact Abelian group and $B(\Gamma, \delta)$ a regular Bohr set. Suppose that $f \in A(G)$ and $\epsilon, \eta \in(0,1]$. Write $A_{f}$ for the quantity $\|f\|_{A(G)}\|f\|_{L^{\infty}(B(\Gamma, \delta))}^{-1}$. Then there is a set $\Lambda$ of characters and a $\delta^{\prime} \in(0,1]$ with

$$
|\Lambda| \ll \epsilon^{-1}\left(1+\log A_{f}\right) \text { and } \delta^{\prime} \gg \epsilon^{2} \eta \delta / d^{2}\left(1+\log A_{f}\right),
$$

such that

$\left\{\gamma \in \widehat{G}:|\widehat{f d \beta}(\gamma)| \geq \epsilon\|f\|_{L^{\infty}(B(\Gamma, \delta))}\right\} \subset\left\{\gamma:|1-\gamma(x)| \leq \eta\right.$ for all $\left.x \in B\left(\Gamma \cup \Lambda, \delta^{\prime}\right)\right\}$.

We do not require Propositions 7.2 or 7.3 in our application and their methods of proof are in both cases simplifications of the method used for Proposition 7.4 so we shall restrict our attention to proving that result.

A key tool in Theorem 2.2 is that of dissociativity; in the local setting we use the following version of it. If $S$ is a non-empty symmetric neighborhood of $0_{\widehat{G}}$ then we say that $\Lambda$ is $S$-dissociated if

$$
m \in\{-1,0,1\}^{\Lambda} \text { and } m . \Lambda \in S \text { implies that } m \equiv 0 .
$$

Vanilla dissociativity corresponds to taking $S=\left\{0_{\widehat{G}}\right\}$, and typically in the local setting $S$ will be a set of characters at which $\widehat{\beta}$ is large for some Bohr set $B(\Gamma, \delta)$.

In the same way as Theorem 2.2 follows from Lemma 3.1 and Proposition 3.2 Proposition 7.4 follows from the next two lemmas.

Lemma 7.5. Suppose that $G$ is a compact Abelian group and $B(\Gamma, \delta)$ is a regular Bohr set. Suppose that $\eta^{\prime}, \eta \in(0,1]$ and $\Delta$ is a set of characters on $G$. If $\Lambda$ is a maximal $\left\{\gamma:|\widehat{\beta}(\gamma)| \geq \eta^{\prime}\right\}$-dissociated subset of $\Delta$ then there is a $\delta^{\prime} \gg$ $\min \left\{\eta /|\Lambda|, \eta^{\prime} \eta \delta / d\right\}$ such that

$$
\Delta \subset\left\{\gamma:|1-\gamma(x)| \leq \eta \text { for all } x \in B\left(\Gamma \cup \Lambda, \delta^{\prime}\right)\right\} .
$$

Lemma 7.6. Suppose that $G$ is a compact Abelian group and $B(\Gamma, \delta)$ is a regular Bohr set. Suppose that $f \in A(G)$ and $\epsilon, \eta \in(0,1]$. Write $A_{f}$ for the quantity $\|f\|_{A(G)}\|f\|_{L^{\infty}(B(\Gamma, \delta))}^{-1}$. Then there is a $\delta^{\prime} \gg \epsilon^{2} \delta / d\left(1+\log A_{f}\right)$ regular for $\Gamma$ such that if $\Lambda$ is a $\left\{\gamma:\left|\widehat{\beta}^{\prime}(\gamma)\right| \geq 1 / 3\right\}$-dissociated subset of $\{\gamma \in \widehat{G}:|\widehat{f d \beta}(\gamma)| \geq$ $\left.\epsilon\|f\|_{L^{\infty}(B(\Gamma, \delta))}\right\}$ then

$$
|\Lambda| \ll \epsilon^{-1}\left(1+\log A_{f}\right) .
$$

7.7. The proof of Lemma 7.5. The following lemma localizes Lemma 3.1, which corresponds to the case $S=\left\{0_{\widehat{G}}\right\}$.

Lemma 7.8. Suppose that $G$ is a compact Abelian group. Suppose that $S$ is a non-empty symmetric neighborhood of $0_{\widehat{G}}$. Suppose that $\Delta$ is a set of characters on $G$ and $\Lambda$ is a maximal $S$-dissociated subset of $\Delta$. Then $\Delta \subset\langle\Lambda\rangle+S$.

Proof. If $\lambda_{0} \in \Delta \backslash(\langle\Lambda\rangle+S)$ then we put $\Lambda^{\prime}:=\Lambda \cup\left\{\lambda_{0}\right\}$, which is a strict superset of $\Lambda$, and a subset of $\Delta$. It turns out that $\Lambda^{\prime}$ is also $S$-dissociated which contradicts the maximality of $\Lambda$. Suppose that $m: \Lambda^{\prime} \rightarrow\{-1,0,1\}$ and $m \cdot \Lambda^{\prime} \in S$. Then we have three possibilities for the value of $m_{\lambda_{0}}$ :

(1) $m . \Lambda^{\prime}=\lambda_{0}+\left.m\right|_{\Lambda} \cdot \Lambda$, in which case $\lambda_{0} \in-\left.m\right|_{\Lambda} \cdot \Lambda+S \subset\langle\Lambda\rangle+S$ - a contradiction; 
(2) $m . \Lambda^{\prime}=-\lambda_{0}+\left.m\right|_{\Lambda} \cdot \Lambda$, in which case $\left.\lambda_{0} \in m\right|_{\Lambda} \cdot \Lambda-S \subset\langle\Lambda\rangle+S$ - a contradiction;

(3) $m . \Lambda^{\prime}=\left.m\right|_{\Lambda} \cdot \Lambda$, in which case $\left.m\right|_{\Lambda} \equiv 0$ since $\Lambda$ is $S$-dissociated and hence $m \equiv 0$.

It follows that $m . \Lambda^{\prime} \in S \Rightarrow m \equiv 0$ i.e. $\Lambda^{\prime}$ is $S$-dissociated as claimed.

Lemma 7.5 then follows from the above and the next lemma.

Lemma 7.9. Suppose that $G$ is a compact Abelian group and $B(\Gamma, \delta)$ is a regular Bohr set. Suppose that $\eta^{\prime}, \eta \in(0,1]$ and $\Lambda$ is a set of characters on $G$. Then there is a $\delta^{\prime} \gg \min \left\{\eta /|\Lambda|, \eta^{\prime} \eta \delta / d\right\}$ such that

$$
\langle\Lambda\rangle+\left\{\gamma:|\widehat{\beta}(\gamma)| \geq \eta^{\prime}\right\} \subset\left\{\gamma:|1-\gamma(x)| \leq \eta \text { for all } x \in B\left(\Gamma \cup \Lambda, \delta^{\prime}\right)\right\} .
$$

Proof. The lemma has two parts.

(1) If $\lambda \in\langle\Lambda\rangle$ then

$$
|1-\lambda(x)| \leq \sum_{\lambda^{\prime} \in \Lambda}\left|1-\lambda^{\prime}(x)\right|
$$

so there is a $\delta^{\prime \prime} \gg \eta /|\Lambda|$ such that

$$
\langle\Lambda\rangle \subset\left\{\gamma:|1-\gamma(x)| \leq \eta / 2 \text { for all } x \in B\left(\Lambda, \delta^{\prime \prime}\right)\right\} .
$$

(2) By Lemma 6.7 there is a $\delta^{\prime \prime \prime} \gg \eta \eta^{\prime} \delta / d$ such that

$$
\left\{\gamma:|\widehat{\beta}(\gamma)| \geq \eta^{\prime}\right\} \subset\left\{\gamma:|1-\gamma(x)| \leq \eta / 2 \text { for all } x \in B\left(\Gamma, \delta^{\prime \prime \prime}\right)\right\} .
$$

Taking $\delta^{\prime}=\min \left\{\delta^{\prime \prime}, \delta^{\prime \prime \prime}\right\}$ we have the result by the triangle inequality.

7.10. The proof of Lemma 7.6. The proof follows that of Proposition 3.2 with the additional ingredient of smoothed measures.

Suppose that $B(\Gamma, \delta)$ is a regular Bohr set on $G$. For $L \in \mathbb{N}$ and $\kappa \in(0,1]$ we write

$$
\tilde{\beta}_{\Gamma, \delta}^{L, \kappa}:=\beta_{\Gamma,(1-\kappa) \delta} * \beta_{\Gamma, \kappa \delta / L}^{L},
$$

where here, as in future, juxtaposition of measures denotes convolution. $\tilde{\beta}_{\Gamma, \delta}^{L, \kappa}$ is a good approximation to $\beta$ in $M(G)$ :

$$
\left\|\tilde{\beta}_{\Gamma, \delta}^{L, \kappa}-\beta_{\Gamma, \delta}\right\| \leq\left\|\beta_{\Gamma, \delta(1-\kappa)} * \mu-\beta_{\Gamma, \delta(1-\kappa)}\right\|+\left\|\beta_{\Gamma, \delta(1-\kappa)}-\beta_{\Gamma, \delta}\right\|
$$

where $\mu=\beta_{\Gamma, \kappa \delta / L}^{L}$, the convolution of $\beta_{\Gamma, \kappa \delta / L}$ with itself $L$ times. We deal with the first term using Corollary 6.5 which yields

$$
\left\|\beta_{\Gamma, \delta(1-\kappa)} * \mu-\beta_{\Gamma, \delta(1-\kappa)}\right\| \ll \kappa d
$$

since supp $\mu \subset B(\Gamma, \kappa \delta)$. For the second term we have

$$
\begin{aligned}
\left\|\beta_{\Gamma, \delta(1-\kappa)}-\beta_{\Gamma, \delta}\right\| & \leq\left\|\beta_{\Gamma, \delta(1-\kappa)}-\left.\beta_{\Gamma, \delta}\right|_{B(\Gamma, \delta(1-\kappa))}\right\|+\left\|\left.\beta_{\Gamma, \delta}\right|_{B(\Gamma, \delta) \backslash B(\Gamma, \delta(1-\kappa))}\right\| \\
& =\left(1-\frac{\mu_{G}(B(\Gamma, \delta(1-\kappa)))}{\mu_{G}(B(\Gamma, \delta))}\right)+\left\|\left.\beta_{\Gamma, \delta}\right|_{B(\Gamma, \delta) \backslash B(\Gamma, \delta(1-\kappa))}\right\| \\
& =O(\kappa d)+\left\|\left.\beta_{\Gamma, \delta}\right|_{B(\Gamma, \delta) \backslash B(\Gamma, \delta(1-\kappa))}\right\| \text { by regularity, } \\
& =O(\kappa d)+\left(\frac{\mu_{G}(B(\Gamma, \delta))-\mu_{G}(B(\Gamma, \delta(1-\kappa)))}{\mu_{G}(B(\Gamma, \delta))}\right) \\
& =O(\kappa d) \text { by regularity. }
\end{aligned}
$$


It follows that $\left\|\tilde{\beta}_{\Gamma, \delta}^{L, \kappa}-\beta\right\|=O(\kappa d)$ and hence if $f \in L^{\infty}(B(\Gamma, \delta))$ we have

$$
\left|\widehat{f d \tilde{\beta}_{\Gamma, \delta}^{L, \kappa}}(\gamma)-\widehat{f d \beta_{\Gamma, \delta}}(\gamma)\right| \ll\|f\|_{L^{\infty}(B(\Gamma, \delta))} \kappa d .
$$

Proof. We begin by fixing $\kappa$ and $L$ in the smoothed measure $\tilde{\beta}_{\Gamma, \delta}^{L, \kappa}$ so that we may dispense with the superscripts and subscripts and simply write $\tilde{\beta}$. Take $L=2 R$, where $R$ will be chosen later and $\kappa \gg \epsilon / d$ so that

$$
\left|\widehat{f d \tilde{\beta}_{\Gamma, \delta}^{L, \kappa}}(\gamma)-\widehat{f d \beta_{\Gamma, \delta}}(\gamma)\right| \leq 2^{-1} \epsilon\|f\|_{L^{\infty}(B(\Gamma, \delta))},
$$

which we may certainly do by (7.1), and also so that $\delta^{\prime}:=\kappa \delta / L$ is regular for $\Gamma$. As usual this last requirement is possible by Proposition 6.3. It follows that

$$
|\widehat{f d \beta}(\gamma)| \geq \epsilon\|f\|_{L^{\infty}(B(\Gamma, \delta))} \Rightarrow|\widehat{f d \tilde{\beta}}(\gamma)| \geq 2^{-1} \epsilon\|f\|_{L^{\infty}(B(\Gamma, \delta))} .
$$

Now suppose that $\Lambda$ is a $\left\{\gamma:\left|\widehat{\beta}^{\prime}(\gamma)\right| \geq 1 / 3\right\}$-dissociated subset of $\{\gamma \in \widehat{G}: \mid \widehat{f d \beta} \gamma) \mid \geq$ $\left.\epsilon\|f\|_{L^{\infty}(B(\Gamma, \delta))}\right\}$ and that $\Lambda^{\prime} \subset \Lambda$ has size at most $R$. $\Lambda^{\prime}$ is certainly still $\{\gamma$ : $\left.\left|\widehat{\beta^{\prime}}(\gamma)\right| \geq 1 / 3\right\}$-dissociated. We define

$$
\omega(\lambda):=\frac{\widehat{f d \tilde{\beta}}(\lambda)}{|\widehat{f d \tilde{\beta}}(\lambda)|} \text { for all } \lambda \in \Lambda^{\prime} .
$$

$\omega \in \ell^{\infty}\left(\Lambda^{\prime}\right),\|\omega\|_{\infty} \leq 1, \Lambda^{\prime}$ is finite and $\Lambda^{\prime}$ is dissociated (since it is $\left\{\gamma:\left|\widehat{\beta}^{\prime}(\gamma)\right| \geq\right.$ $1 / 3\}$-dissociated) so we may apply Proposition 3.3 to get the auxiliary measure $\mu_{\eta}$. To leverage the stronger dissociativity condition we introduce a Riesz product:

$$
q:=\prod_{\lambda \in \Lambda^{\prime}}\left(1+\frac{\lambda+\bar{\lambda}}{2}\right) .
$$

Recall (from 4 if necessary) that $q$ is non-negative and since $\Lambda^{\prime}$ is dissociated $\|q\|_{1}=1$.

Plancherel's theorem gives

$$
\left\langle f d \tilde{\beta}, \mu_{\eta} * q\right\rangle=\sum_{\gamma \in \widehat{G}} \widehat{f d \tilde{\beta}}(\gamma) \overline{\widehat{q}(\gamma) \widehat{\mu_{\eta}}(\gamma)} .
$$

We begin by bounding the right hand side from below using the bound on $\left|\widehat{\mu_{\eta}}(\lambda)\right|$ for $\lambda \notin \widehat{G}$ and the fact that $\widehat{q}(\lambda) \geq 1 / 2$ if $\lambda \in \Lambda^{\prime}$.

$$
\begin{aligned}
\left|\sum_{\gamma \in \widehat{G}} \widehat{f d \tilde{\beta}}(\gamma) \overline{\widehat{q}(\gamma) \widehat{\mu_{\eta}}(\gamma)}\right| & =\mid \sum_{\lambda \in \Lambda^{\prime}} \widehat{f d \tilde{\beta}}(\lambda) \overline{\widehat{q}(\lambda) \widehat{\mu_{\eta}}(\lambda)}+\sum_{\lambda \notin \Lambda^{\prime}} \widehat{f d \tilde{\beta}}(\lambda) \overline{\widehat{q}(\lambda) \widehat{\mu_{\eta}}(\lambda) \mid} \\
& \geq\left|\sum_{\lambda \in \Lambda^{\prime}} \widehat{f d \tilde{\beta}}(\lambda) \widehat{\widehat{q}(\lambda) \widehat{\mu_{\eta}}(\lambda)}\right|-\left|\sum_{\lambda \notin \Lambda^{\prime}} \widehat{f d \tilde{\beta}}(\lambda) \overline{\widehat{q}(\lambda) \widehat{\mu_{\eta}}(\lambda)}\right| \\
& \geq\left|\sum_{\lambda \in \Lambda^{\prime}} \widehat{f d \tilde{\beta}}(\lambda) \overline{\widehat{q}(\lambda) \omega(\lambda)}\right|-\eta \sum_{\lambda \in \widehat{G}}|\widehat{q}(\lambda)||\widehat{f d \tilde{\beta}}(\lambda)| \\
& \geq 2^{-1} \sum_{\lambda \in \Lambda^{\prime}}|\widehat{f d \tilde{\beta}}(\lambda)|-\eta \sum_{\lambda \in \widehat{G}} \sum_{\gamma \in \widehat{G}}|\widehat{q}(\lambda)||\widehat{f}(\gamma) \widehat{\tilde{\beta}}(\lambda-\gamma)| \\
& \geq 2^{-1} \sum_{\lambda \in \Lambda^{\prime}}|\widehat{f d \tilde{\beta}}(\lambda)|-\eta\|f\|_{A(G)} \sup _{\gamma \in \widehat{G}} \sum_{\lambda \in \widehat{G}}|\widehat{q}(\lambda)||\widehat{\tilde{\beta}}(\lambda-\gamma)| .
\end{aligned}
$$


T. SANDERS

For any $\gamma \in \widehat{G}$ we can estimate the last sum in a manner independent of $\gamma$ by using a positivity argument:

$$
\begin{aligned}
\sum_{\lambda \in \widehat{G}}|\widehat{q}(\lambda)||\widetilde{\tilde{\beta}}(\lambda-\gamma)| & =\sum_{\lambda \in \widehat{G}}|\widehat{q}(\gamma-\lambda)||\widehat{\tilde{\beta}}(\lambda)| \text { by symmetry of } \widehat{\tilde{\beta}}, \\
& =\sum_{\lambda \in \widehat{G}}|\widehat{q}(\gamma-\lambda)|\left|\widehat{\beta}(\lambda) \widehat{\beta}^{\prime}(\lambda)^{L}\right| \text { by definition of } \widehat{\tilde{\beta}}, \\
& \leq \sum_{\lambda \in \widehat{G}}|\widehat{q}(\gamma-\lambda)|\left|\widehat{\beta^{\prime}}(\lambda)\right|^{L} \text { since }|\widehat{\beta}(\lambda)| \leq\|\beta\|=1, \\
& =\widehat{q d \beta^{\prime L}}(\gamma) \text { since } L \text { is even and } \widehat{q} \geq 0, \\
& \leq\left\|q d \beta^{L}\right\| \\
& =\widehat{q d \beta^{\prime L}}\left(0_{\widehat{G}}\right) \text { by non-negativity of } q d \beta^{\prime L}, \\
& =\sum_{\lambda \in \widehat{G}} \widehat{q}(\lambda)\left|\widehat{\beta}^{\prime}(\lambda)\right|^{L} \text { by symmetry of } \widehat{q} .
\end{aligned}
$$

We estimate this by splitting the range of summation into two parts:

$$
\sum_{\lambda \in \widehat{G}} \widehat{q}(\lambda)\left|\widehat{\beta}^{\prime}(\lambda)\right|^{L} \leq \sum_{\lambda:\left|\widehat{\beta}^{\prime}(\lambda)\right| \geq 1 / 3} \widehat{q}(\lambda)\left|\widehat{\beta}^{\prime}(\lambda)\right|^{L}+\sum_{\lambda:\left|\widehat{\beta}^{\prime}(\lambda)\right| \leq 1 / 3} \widehat{q}(\lambda)\left|\widehat{\beta}^{\prime}(\lambda)\right|^{L} .
$$

(1) For the first sum: $|\widehat{q}(\lambda)| \leq\|q\|_{1}=1$ and $\left|\widehat{\beta}^{\prime}(\lambda)^{L}\right| \leq\left\|\beta^{\prime L}\right\|=1$ so that each summand is at most 1 , furthermore supp $\widehat{q} \subset\left\langle\Lambda^{\prime}\right\rangle$ so

$$
\sum_{\lambda:\left|\widehat{\beta^{\prime}}(\lambda)\right| \geq 1 / 3} \widehat{q}(\lambda)\left|\widehat{\beta}^{\prime}(\lambda)\right|^{L} \leq \sum_{\lambda \in\left\langle\Lambda^{\prime}\right\rangle:\left|\widehat{\beta}^{\prime}(\lambda)\right| \geq 1 / 3} 1 .
$$

This range of summation contains at most 1 element by $\left\{\gamma:\left|\widehat{\beta}^{\prime}(\gamma)\right| \geq 1 / 3\right\}$ dissociativity of $\Lambda^{\prime}$, and hence the sum is bounded above by 1 .

(2) For the second sum: $|\widehat{q}(\lambda)| \leq\|q\|_{1}=1$ and $\left|\widehat{\beta}^{\prime}(\lambda)^{L}\right| \leq 3^{-L}$ for $\lambda$ in the range of summation so that each summand is at most $9^{-\left|\Lambda^{\prime}\right|}$, however supp $\widehat{q} \subset$ $\left\langle\Lambda^{\prime}\right\rangle$ and $\left|\left\langle\Lambda^{\prime}\right\rangle\right| \leq 3^{\left|\Lambda^{\prime}\right|}$ so

$$
\sum_{\lambda:\left|\widehat{\beta}^{\prime}(\lambda)\right| \leq 1 / 3} \widehat{q}(\lambda)\left|\widehat{\beta}^{\prime}(\lambda)\right|^{L} \leq \sum_{\lambda \in\left\langle\Lambda^{\prime}\right\rangle} 9^{-\left|\Lambda^{\prime}\right|} \leq 1 .
$$

It follows that the right hand side of (7.2) is bounded above by 2, and working backwards these estimates combine to show that

$$
\sum_{\lambda \in \widehat{G}}|\widehat{q}(\lambda)||\widetilde{\tilde{\beta}}(\lambda-\gamma)| \leq 2 \text { for all } \gamma \in \widehat{G}
$$

and hence that

$$
\left|\left\langle f d \tilde{\beta}, \mu_{\eta} * q\right\rangle\right| \geq 2^{-1} \sum_{\lambda \in \Lambda^{\prime}}|\widehat{f d \tilde{\beta}}(\lambda)|-2 \eta\|f\|_{A(G)} .
$$

To estimate the inner product from above we have:

$$
\left|\left\langle f d \tilde{\beta}, \mu_{\eta} * q\right\rangle\right| \leq\|f\|_{L^{\infty}(B(\Gamma, \delta))}\|\tilde{\beta}\|\left\|\mu_{\eta}\right\|\|q\|_{1} \ll\|f\|_{L^{\infty}(B(\Gamma, \delta))}\left(1+\log \eta^{-1}\right)
$$

by the estimate for $\left\|\mu_{\eta}\right\|$ given in Proposition 3.3. Combining this with our lower bound for the inner product in (7.3) and the fact that if $\lambda \in \Lambda^{\prime}$ then $|\widehat{f d \tilde{\beta}}(\lambda)| \geq$ 
$2^{-1} \epsilon\|f\|_{L^{\infty}(B(\Gamma, \delta))}$ gives

$$
\|f\|_{L^{\infty}(B(\Gamma, \delta))}\left(1+\log \eta^{-1}\right)+\eta\|f\|_{A(G)} \gg\left|\Lambda^{\prime}\right| \epsilon\|f\|_{L^{\infty}(B(\Gamma, \delta))} .
$$

Choosing $\eta^{-1}=\|f\|_{A(G)}\|f\|_{L^{\infty}(B(\Gamma, \delta))}^{-1}$ yields that

$$
\left|\Lambda^{\prime}\right| \ll \epsilon^{-1}\left(1+\log A_{f}\right) .
$$

Let $C$ be the absolute constant implicit in the notation on the right so that $\left|\Lambda^{\prime}\right| \leq$ $C \epsilon^{-1}\left(1+\log A_{f}\right)$ is always true, and set $R:=\left\lceil C \epsilon^{-1}\left(1+\log A_{f}\right)\right\rceil+1$. If $|\Lambda|$ is a

$\left\{\gamma:\left|\widehat{\beta}^{\prime}(\gamma)\right| \geq 1 / 3\right\}$-dissociated set of size greater than $R$, then let $\Lambda^{\prime}$ be a subset of $\Lambda$ of size $R$, which is automatically $\left\{\gamma:\left|\widehat{\beta}^{\prime}(\gamma)\right| \geq 1 / 3\right\}$-dissociated because $\Lambda$ is $\left\{\gamma:\left|\widehat{\beta}^{\prime}(\gamma)\right| \geq 1 / 3\right\}$-dissociated. By the above

$$
C \epsilon^{-1}\left(1+\log A_{f}\right)<\left\lceil C \epsilon^{-1}\left(1+\log A_{f}\right)\right\rceil+1=R=\left|\Lambda^{\prime}\right| \leq C \epsilon^{-1}\left(1+\log A_{f}\right),
$$

which is a contradiction and hence if $\Lambda$ is $\left\{\gamma:\left|\widehat{\beta}^{\prime}(\gamma)\right| \geq 1 / 3\right\}$-dissociated then $|\Lambda|<R \ll \epsilon^{-1}\left(1+\log A_{f}\right)$ as required.

\section{An introduction to Littlewood's Problem}

Finally we turn to addressing the problem announced in the introduction, but although the contents of $\$ 7$ may be taken as a black box for the purposes of the following sections, 96 is a necessary notational prerequisite.

It is natural to begin by considering a qualitative analogue of our problem, in particular we shall start by proving the following well known result.

Proposition 8.1. (Qualitative Littlewood problem) Suppose that $A \subset \mathbb{T}$ has density $\alpha$ with $0<\alpha<1$. Then $\chi_{A} \notin A(\mathbb{T})$.

The proof of this proceeds in three stages, the first two of which are naturally set in an arbitrary compact Abelian group $G$.

(1) (Fourier inversion) First, if $f \in A(G)$ then we may define the function

$$
\widetilde{f}(x):=\sum_{\gamma \in \widehat{G}} \widehat{f}(\gamma) \gamma(x),
$$

which is continuous since it is the uniform limit of continuous functions. The Fourier inversion theorem tells us that $\|\widetilde{f}-f\|_{L^{\infty}(G)}=0$.

(2) (Averaging) Secondly, by averaging there are elements $x_{0}, x_{1} \in G$ such that

$$
\widetilde{f}\left(x_{0}\right) \leq \int f d \mu_{G} \leq \widetilde{f}\left(x_{1}\right),
$$

since $\int f d \mu_{G}=\int \widetilde{f} d \mu_{G}$.

(3) (Intermediate value theorem) Finally we suppose (for a contradiction) that $\chi_{A} \in A(\mathbb{T})$ so that by the intermediate value theorem there is some $x \in \mathbb{T}$ such that $\widetilde{\chi_{A}}(x)=\alpha$. Continuity ensures that there is an open ball $x+B$ on which $\widetilde{\chi_{A}}$ is very close to $\alpha$, and in particular, since $\alpha \in(0,1)$, on which $\widetilde{\chi_{A}}$ only takes values in $(0,1)$. Since $\left\|\chi_{A}-\widetilde{\chi_{A}}\right\|_{L^{\infty}(G)}=0$ and $\mu(x+B)>0$ it follows that $\chi_{A}$ equals $\widetilde{\chi_{A}}$ for some point in $x+B$, but this contradicts the fact that $\chi_{A}$ can only take the values 0 or 1 . 
If we try to transfer this argument to $G=\mathbb{Z} / p \mathbb{Z}$ it breaks down at the third stage when we apply the intermediate value theorem. It is easy enough to remedy this and prove a sensible discrete analogue of the intermediate value theorem; the following, for example, is in [GK09.

Proposition 8.2. (Discrete intermediate value theorem) Suppose that $p$ is prime number. Suppose that $f: \mathbb{Z} / p \mathbb{Z} \rightarrow \mathbb{R}$ and that there is some non-zero $y \in \mathbb{Z} / p \mathbb{Z}$ such that

$$
|f(x+y)-f(x)| \leq \epsilon\|f\|_{\infty} \text { for all } x \in \mathbb{Z} / p \mathbb{Z} .
$$

Then there is some $x \in \mathbb{Z} / p \mathbb{Z}$ such that

$$
\left|f(x)-\int f d \mu_{\mathbb{Z} / p \mathbb{Z}}\right| \leq 2^{-1} \epsilon\|f\|_{\infty} .
$$

Of course this has only moved the difficulty: to use this result we need to replace the continuity in the first stage of our argument with the sort of quantitative continuity used in this proposition.

It turns out that we already have a ready supply of functions which are continuous in this new sense: Suppose that $f \in L^{\infty}(G)$ and $B(\Gamma, \delta)$ is a regular Bohr set. Then by Corollary 6.6 we may pick $\delta^{\prime} \gg \epsilon \delta / d$ such that

$$
\|f * \beta-f * \beta(x)\|_{L^{\infty}\left(x+B\left(\Gamma, \delta^{\prime}\right)\right)} \leq \epsilon\|f\|_{L^{\infty}(G)} .
$$

Now if $\mu_{G}\left(B\left(\Gamma, \delta^{\prime}\right)\right)>p^{-1}$ then $B\left(\Gamma, \delta^{\prime}\right)$ has a non-identity element and hence the discrete intermediate value theorem applies.

Essentially the same argument which shows that if $f \in A(G)$ then $\|f-\widetilde{f}\|_{L^{\infty}(G)}=$ 0 for some continuous function $\widetilde{f}$, can be made quantitative to show that there is a regular Bohr set $B(\Gamma, \delta)$ such that $\|f-f * \beta\|_{L^{\infty}(G)}$ is small and, by our previous observations, $f * \beta$ is quantitatively continuous.

To be concrete suppose that $G$ is a compact Abelian group, $f \in A(G)$ and write $A_{f}:=\|f\|_{A(G)}\|f\|_{L^{\infty}(G)}^{-1}$. Then there is a finite set of characters $\Gamma$ such that

$$
\sum_{\gamma \notin \Gamma}|\widehat{f}(\gamma)| \leq \epsilon A_{f}^{-1}\|f\|_{A(G)} / 3 .
$$

Pick $\delta \gg \epsilon A_{f}^{-1}$ such that

$$
B(\Gamma, \delta) \subset\left\{x \in G:|1-\gamma(x)| \leq \epsilon A_{f}^{-1} / 3 \text { for all } \gamma \in \Gamma\right\},
$$

and such that $\delta$ is regular for $\Gamma$ by Proposition 6.3. It is easy to see that

$$
|1-\widehat{\beta}(\gamma)| \leq \epsilon A_{f}^{-1} / 3 \text { if } \gamma \in \Gamma,
$$

and it follows that

$$
\begin{aligned}
\|f-f * \beta\|_{L^{\infty}(G)} & \leq \sum_{\gamma \in \widehat{G}}|1-\widehat{\beta}(\gamma)||\widehat{f}(\gamma)| \\
& \leq \sum_{\gamma \in \Gamma}|1-\widehat{\beta}(\gamma)||\widehat{f}(\gamma)|+\sum_{\gamma \notin \Gamma}|1-\widehat{\beta}(\gamma)||\widehat{f}(\gamma)| \\
& \leq\left(\epsilon A_{f}^{-1} / 3\right) \sum_{\gamma \in \widehat{G}}|\widehat{f}(\gamma)|+2 \sum_{\gamma \notin \Gamma}|\widehat{f}(\gamma)| \\
& \leq \epsilon A_{f}^{-1}\|f\|_{A(G)}=\epsilon\|f\|_{L^{\infty}(G)} .
\end{aligned}
$$

In slightly formal language this has proved the following result. 
Theorem 8.3. Suppose that $G$ is a compact Abelian group, $f \in A(G)$ and $\epsilon \in(0,1]$. Write $A_{f}$ for the quantity $\|f\|_{A(G)}\|f\|_{L^{\infty}(G)}^{-1}$. Then there is a Bohr set $B(\Gamma, \delta)$ with

$$
d<\infty \text { and } \delta^{-1} \ll \epsilon^{-1} A_{f},
$$

and a narrower Bohr set $B\left(\Gamma, \delta^{\prime}\right)$ with $\delta^{\prime} \gg \epsilon \delta / d$ such that

$$
\sup _{x \in G}\|f * \beta-f * \beta(x)\|_{L^{\infty}\left(x+B\left(\Gamma, \delta^{\prime}\right)\right)} \leq \epsilon\|f\|_{L^{\infty}(G)}
$$

and

$$
\sup _{x \in G}\|f-f * \beta\|_{L^{\infty}\left(x+B\left(\Gamma, \delta^{\prime}\right)\right)} \leq \epsilon\|f\|_{L^{\infty}(G)} .
$$

Of course, as we observed before, this is only useful to us if $B\left(\Gamma, \delta^{\prime}\right)$ contains a non-zero element. We can use Lemma 6.2 to estimate its size: If $G=\mathbb{Z} / p \mathbb{Z}$, then $B\left(\Gamma, \delta^{\prime}\right)$ contains a non-zero element if

$$
\left(c \epsilon^{2} A_{f}^{-1} / d\right)^{d}>p^{-1} \text { for some absolute } c>0 .
$$

Unfortunately, because we have no control over $d$, we have no way of ensuring this inequality. The content of this paper can be seen as an effort to make this method work by getting control of $d$; the main result is the following refinement of Theorem 8.3 .

Theorem 8.4. Suppose that $G$ is a compact Abelian group, $f \in A(G)$ and $\epsilon \in(0,1]$. Write $A_{f}$ for the quantity $\|f\|_{A(G)}\|f\|_{\infty}^{-1}$. Then there is a Bohr set $B(\Gamma, \delta)$ with

$$
d \ll \epsilon^{-2} A_{f}\left(1+\log A_{f}\right)\left(1+\log \epsilon^{-1} A_{f}\right) \text { and } \log \delta^{-1} \ll \epsilon^{-2} A_{f}\left(1+\log \epsilon^{-1} A_{f}\right),
$$

and a narrower Bohr set $B\left(\Gamma, \delta^{\prime}\right)$ with $\delta^{\prime} \gg \epsilon \delta / d$ such that

$$
\sup _{x \in G}\|f * \beta-f * \beta(x)\|_{L^{\infty}\left(x+B\left(\Gamma, \delta^{\prime}\right)\right)} \leq \epsilon\|f\|_{L^{\infty}(G)}
$$

and

$$
\sup _{x \in G}\|f-f * \beta\|_{L^{2}\left(x+B\left(\Gamma, \delta^{\prime}\right)\right)} \leq \epsilon\|f\|_{L^{\infty}(G)} .
$$

Note that to gain control of $d$ we have had to sacrifice some control of $\delta$ and of the error in approximating $f$ by $f * \beta$.

There are now three remaining sections to the paper.

- 99 details our arguments in the model setting of $\mathbb{F}_{2}^{n}$.

- $\$ 10$ proves Theorem 8.4 following the outline of $\$ 9$ and using the tools of $\S 9 \sqrt[6]{6} 7$

- Finally 11 completes the proof of Theorem 1.4 and concludes with some remarks and a conjecture.

\section{The ARgument in A MOdel SETting}

Many proofs of results for compact Abelian groups can be modelled much more cleanly in $\mathbb{F}_{2}^{n}$, indeed we have already partially seen this fact in 4 , and so the purpose of this section is to prove the following result, which is the model version of the Theorem 8.4.

Theorem 9.1. Suppose that $G=\mathbb{F}_{2}^{n}$. Suppose that $f \in A(G)$ and $\epsilon \in(0,1]$. Write $A_{f}$ for the quantity $\|f\|_{A(G)}\|f\|_{L^{\infty}(G)}^{-1}$. Then there is a subspace $V$ of $G$ with

$$
\operatorname{codim} V \ll \epsilon^{-2} A_{f}\left(1+\log A_{f}\right)\left(1+\log \epsilon^{-1} A_{f}\right),
$$


and

$$
\sup _{x \in G}\left\|f-f * \mu_{V}\right\|_{L^{2}(x+V)} \leq \epsilon\|f\|_{L^{\infty}(G)} .
$$

The first part of the conclusion of Theorem 8.4 is unnecessary since $f * \mu_{V}$ is constant on cosets of $V$ and hence

$$
\sup _{x \in G}\left\|f * \mu_{V}-f * \mu_{V}(x)\right\|_{L^{\infty}(x+V)}=0 .
$$

9.2. The basic quantitative argument. We begin with an argument which proves the following weak version of Theorem 9.1 the argument will form the basis of our proof of that theorem.

Theorem 9.3. Suppose that $G=\mathbb{F}_{2}^{n}$. Suppose that $f \in A(G)$ and $\epsilon \in(0,1]$. Write $A_{f}$ for the quantity $\|f\|_{A(G)}\|f\|_{L^{\infty}(G)}^{-1}$. Then there is a subspace $V$ of $G$ with

$$
\operatorname{codim} V \leq 2^{3} \epsilon^{-4} A_{f}^{3}
$$

and

$$
\sup _{x \in G}\left\|f-f * \mu_{V}\right\|_{L^{2}(x+V)} \leq \epsilon\|f\|_{L^{\infty}(G)} .
$$

The technique is iterative; the driving component is the following lemma.

Lemma 9.4. (Iteration lemma 1) Suppose that $G=\mathbb{F}_{2}^{n}$ and $\Gamma^{\perp}$ is an annihilator in $G$. Suppose that $f \in A(G)$ and $\epsilon \in(0,1]$. Write $A_{f}$ for the quantity $\|f\|_{A(G)}\|f\|_{L^{\infty}(G)}^{-1}$. Then at least one of the following is true.

(1) ( $f$ is close to a continuous function)

$$
\sup _{x \in G}\left\|f-f * \mu_{\Gamma^{\perp}}\right\|_{L^{2}\left(x+\Gamma^{\perp}\right)} \leq \epsilon\|f\|_{L^{\infty}(G)} .
$$

(2) There is a set of characters $\Lambda$ with $|\Lambda| \leq 2 \epsilon^{-2} A_{f}^{2}$ such that

$$
\sum_{\gamma \in(\Gamma \cup \Lambda) \perp \perp}|\widehat{f}(\gamma)|-\sum_{\gamma \in \Gamma^{\perp \perp}}|\widehat{f}(\gamma)| \geq 2^{-2} \epsilon^{2}\|f\|_{L^{\infty}(G)} .
$$

Essentially this says that if $f$ doesn't satisfy the conclusion of Theorem 9.3 for some annihilator $\Gamma^{\perp}$ then there is a smaller annihilator $\Gamma^{\perp}$, which is not too much smaller, that supports more $A(G)$-norm of $f$.

To control the size of $\Gamma^{\perp}$ we use Proposition 7.2 from 97 , in $\mathbb{F}_{2}^{n}$ its statement is particularly simple:

Proposition 9.5. (Model analogue of Proposition 7.2) Suppose that $G=\mathbb{F}_{2}^{n}$ and $\Gamma^{\perp}$ is an annihilator in $G$. Suppose that $f \in A\left(\Gamma^{\perp}\right)$ and $\epsilon \in(0,1]$. Then there is a set $\Lambda$ of characters with

$$
|\Lambda| \leq \epsilon^{-1}\|f\|_{A\left(\Gamma^{\perp}\right)}\|f\|_{L^{\infty}\left(\Gamma^{\perp}\right)}^{-1}
$$

such that

$$
\left\{\gamma \in \widehat{G}:\left|\widehat{f d \mu_{\Gamma^{\perp}}}(\gamma)\right| \geq \epsilon\|f\|_{L^{\infty}\left(\Gamma^{\perp}\right)}\right\} \subset(\Gamma \cup \Lambda)^{\perp \perp} .
$$

Proof of Lemma 9.4. Suppose that

$$
\sup _{x \in G}\left\|f-f * \mu_{\Gamma^{\perp}}\right\|_{L^{2}\left(x+\Gamma^{\perp}\right)}>\epsilon\|f\|_{L^{\infty}(G)} .
$$

Then there is some $x^{\prime} \in G$ which, without loss of generality, is equal to $0_{G}$ such that

$$
\left\|f-f * \mu_{\Gamma^{\perp}}\right\|_{L^{2}\left(x^{\prime}+\Gamma^{\perp}\right)} \geq \epsilon\|f\|_{L^{\infty}(G)} .
$$


For ease of notation write $g=f-f * \mu_{\Gamma^{\perp}}$, and observe that $g$ satisfies the inequalities

$$
\|g\|_{A(G)} \leq\|f\|_{A(G)} \text { and }\|g\|_{L^{\infty}\left(\Gamma^{\perp}\right)} \leq 2\|f\|_{L^{\infty}(G)} .
$$

To see the first of these note that

$$
\|g\|_{A(G)}=\sum_{\gamma \in \widehat{G}}\left|1-\widehat{\mu_{\Gamma^{\perp}}}(\gamma)\right||\widehat{f}(\gamma)| \leq \sup _{\gamma \in \widehat{G}}\left|1-\widehat{\mu_{\Gamma^{\perp}}}(\gamma)\right|\|f\|_{A(G)} \leq\|f\|_{A(G)},
$$

and for the second

$$
\|g\|_{L^{\infty}\left(\Gamma^{\perp}\right)} \leq\|g\|_{L^{\infty}(G)} \leq\|f\|_{L^{\infty}(G)}+\left\|f * \mu_{\Gamma^{\perp}}\right\|_{L^{\infty}(G)} \leq 2\|f\|_{L^{\infty}(G)} .
$$

Returning to (9.1) we have

$$
\begin{aligned}
\epsilon^{2}\|f\|_{L^{\infty}(G)}^{2} & \leq\|g\|_{L^{2}\left(\Gamma^{\perp}\right)}^{2} \\
& =\sum_{\gamma \in \widehat{G}} \widehat{g d \mu_{\Gamma^{\perp}}}(\gamma) \overline{\widehat{g}(\gamma)} \text { by Plancherel's theorem, } \\
& \leq \sum_{\gamma \in \widehat{G}}\left|\widehat{g d \mu_{\Gamma^{\perp}}}(\gamma)\right| \widehat{g}(\gamma) \mid \text { by the triangle inequality. }
\end{aligned}
$$

The characters supporting large values of $\widehat{g d \mu_{\Gamma^{\perp}}}$ make the principal contribution to this sum. Specifically put

$$
\mathcal{C}:=\left\{\gamma \in \widehat{G}:\left|\widehat{g d \mu_{\Gamma^{\perp}}}(\gamma)\right|>\epsilon^{\prime}\|g\|_{L^{\infty}\left(\Gamma^{\perp}\right)}\right\}
$$

where

$$
\epsilon^{\prime}:=2^{-1} \epsilon^{2} A_{f}^{-1}\|f\|_{L^{\infty}(G)}\|g\|_{L^{\infty}\left(\Gamma^{\perp}\right)}^{-1}
$$

Then

$$
\begin{aligned}
\sum_{\gamma \notin \mathcal{C}}\left|\widehat{g d \mu_{\Gamma^{\perp}}}(\gamma) \| \widehat{g}(\gamma)\right| & \leq 2^{-1} \epsilon^{2} A_{f}^{-1}\|f\|_{L^{\infty}(G)} \sum_{\gamma \notin \mathcal{C}}|\widehat{g}(\gamma)| \\
& \leq 2^{-1} \epsilon^{2} A_{f}^{-1}\|f\|_{L^{\infty}(G)}\|g\|_{A(G)} \\
& \leq 2^{-1} \epsilon^{2}\|f\|_{L^{\infty}(G)}^{2} \text { since }\|g\|_{A(G)} \leq\|f\|_{A(G)}
\end{aligned}
$$

Substituting this into (9.2) we conclude that

$$
\sum_{\gamma \in \mathcal{C}}\left|\widehat{g d \mu_{\Gamma^{\perp}}}(\gamma)\right||\widehat{g}(\gamma)| \geq 2^{-1} \epsilon^{2}\|f\|_{L^{\infty}(G)}^{2}
$$

Now certainly $\left|\widehat{g d \mu_{\Gamma^{\perp}}}(\gamma)\right| \leq 2\|f\|_{L^{\infty}(G)}$ so that

$$
2^{-2} \epsilon^{2}\|f\|_{L^{\infty}(G)} \leq \sum_{\gamma \in \mathcal{C}}|\widehat{g}(\gamma)| .
$$

Since $\|g\|_{A\left(\Gamma^{\perp}\right)} \leq\|f\|_{A(G)}$ we may apply Proposition 9.5 to $\mathcal{C}$ to get a set of characters $\Lambda$ with

$$
|\Lambda|<\left(\epsilon^{\prime}\right)^{-1}\|g\|_{A\left(\Gamma^{\perp}\right)}\|g\|_{L^{\infty}\left(\Gamma^{\perp}\right)}^{-1} \leq 2 \epsilon^{-2} A_{f}^{2},
$$

such that $\mathcal{C} \subset(\Gamma \cup \Lambda)^{\perp \perp}$. The lemma follows.

We are now in a position to iterate this and prove Theorem 9.3 . 
Proof of Theorem 9.3. We construct a sequence of annihilators $\Gamma_{k}^{\perp}$ iteratively. Define

$$
L_{k}:=\sum_{\gamma \in \Gamma_{k}^{\perp \perp}}|\widehat{f}(\gamma)|,
$$

and initiate the iteration with $\Gamma_{0}:=\left\{0_{\widehat{G}}\right\}$.

Suppose that we are at stage $k$ of the iteration. Apply the iteration lemma (Lemma 9.4). If we are in the first case of the lemma then put $V=\Gamma_{k}^{\perp}$ and terminate; if not then we get a set of characters $\Lambda$ and put $\Gamma_{k+1}=\Gamma_{k} \cup \Lambda$. It follows from the properties of $\Lambda$ that

$$
\left|\Gamma_{k+1}\right| \leq\left|\Gamma_{k}\right|+2 \epsilon^{-2} A_{f}^{2} \text { and } L_{k+1}-L_{k} \geq 2^{-2} \epsilon^{2}\|f\|_{L^{\infty}(G)} .
$$

By induction we have that after $k$ iterations

$$
\left|\Gamma_{k}\right| \leq k .2 \epsilon^{-2} A_{f}^{2} \text { and } L_{k} \geq k .2^{-2} \epsilon^{2}\|f\|_{L^{\infty}(G)} .
$$

Since $L_{k} \leq\|f\|_{A(G)}$ we conclude that the iteration terminates and

$$
\left|\Gamma_{k}\right| \leq 2^{3} \epsilon^{-4} A_{f}^{3} \text {. }
$$

The theorem follows.

9.6. Refining the basic argument: the proof of Theorem 9.1, To achieve the result in Theorem 9.1 we make two important improvements to the iteration lemma (Lemma 9.4) of the previous argument.

- (Dyadic decomposition) Our first improvement is the observation that having derived (9.3):

$$
\sum_{\gamma \in \mathcal{C}}\left|\widehat{g d \mu_{\Gamma^{\perp}}}(\gamma)\right||\widehat{g}(\gamma)| \geq 2^{-1} \epsilon^{2}\|f\|_{L^{\infty}(G)}^{2}
$$

we can do something better that simply adding all the characters in $\mathcal{C}$ to $\Gamma$. Partition the characters in $\mathcal{C}$ by dyadically decomposing the range of values of $\left|\widehat{g d \mu_{\Gamma^{\perp}}}\right|$ and pick the characters in a dyadic class contributing maximal mass to (9.3). The $A(G)$-norm of $f$ supported on this class is more closely related to the size of $\mathcal{C}$ which yields the first improvement.

- (Structure theorem for the Fourier spectrum) The second improvement replaces the application of Proposition 9.5 with the stronger Proposition 7.3 . which in the model setting has the following simpler statement.

Proposition 9.7. (Model analogue of Proposition 7.3) Suppose that $G=$ $\mathbb{F}_{2}^{n}$ and $\Gamma^{\perp}$ is an annihilator in $G$. Suppose that $f \in A\left(\Gamma^{\perp}\right)$ and $\epsilon \in(0,1]$. Write $A_{f}$ for the quantity $\|f\|_{A\left(\Gamma^{\perp}\right)}\|f\|_{L^{\infty}\left(\Gamma^{\perp}\right)}^{-1}$. Then there is a set $\Lambda$ of characters with $|\Lambda| \ll \epsilon^{-1}\left(1+\log A_{f}\right)$ such that

$$
\left\{\gamma \in \widehat{G}:\left|\widehat{f d \mu_{\Gamma^{\perp}}}(\gamma)\right| \geq \epsilon\|f\|_{L^{\infty}\left(\Gamma^{\perp}\right)}\right\} \subset(\Gamma \cup \Lambda)^{\perp \perp} .
$$

By implementing these two refinements we prove the following iteration lemma.

Lemma 9.8. (Iteration lemma 2) Suppose that $G=\mathbb{F}_{2}^{n}$ and $\Gamma^{\perp}$ is an annihilator in $G$. Suppose that $f \in A(G)$ and $\epsilon \in(0,1]$. Write $A_{f}$ for the quantity $\|f\|_{A(G)}\|f\|_{L^{\infty}(G)}^{-1}$. Then at least one of the following is true.

(1) ( $f$ is close to a continuous function)

$$
\sup _{x \in G}\left\|f-f * \mu_{\Gamma^{\perp}}\right\|_{L^{2}\left(x+\Gamma^{\perp}\right)} \leq \epsilon\|f\|_{L^{\infty}(G)} .
$$


(2) There is a set of characters $\Lambda$ and a non-negative integer $s$ with $|\Lambda| \ll$ $2^{s}\left(1+\log A_{f}\right)$ such that

$$
\sum_{\gamma \in(\Gamma \cup \Lambda) \perp \perp}|\widehat{f}(\gamma)|-\sum_{\gamma \in \Gamma^{\perp \perp}}|\widehat{f}(\gamma)| \gg \frac{2^{s} \epsilon^{2}\|f\|_{L^{\infty}(G)}}{1+\log \epsilon^{-1} A_{f}} .
$$

Proof. We proceed as in the proof of Lemma 9.4 up to the point where we conclude that

$$
\sum_{\gamma \in \mathcal{C}}\left|\widehat{g d \mu_{\Gamma^{\perp}}}(\gamma)\right||\widehat{g}(\gamma)| \geq 2^{-1} \epsilon^{2}\|f\|_{L^{\infty}(G)}^{2}
$$

Write $I_{s}:=\left(2^{-s}\|f\|_{L^{\infty}(G)}, 2^{-(s-1)}\|f\|_{L^{\infty}(G)}\right]$ and partition $\mathcal{C}$ into the sets

$$
\mathcal{C}_{s}:=\left\{\gamma \in \mathcal{C}:\left|\widehat{g d \mu_{\Gamma^{\perp}}}(\gamma)\right| \in I_{s}\right\} \text { for } 0 \leq s \leq 2+\log _{2} \epsilon^{-2} A_{f} .
$$

Note that $\left\{\mathcal{C}_{s}: 0 \leq s \leq 2+\log _{2} \epsilon^{-2} A_{f}\right\}$ covers $\mathcal{C}$ since

$$
\sup _{\gamma \in \mathcal{C}}\left|\widehat{g d \mu_{\Gamma^{\perp}}}(\gamma)\right| \leq \sup _{\gamma \in \widehat{G}}\left|\widehat{g d \mu_{\Gamma^{\perp}}}(\gamma)\right| \leq\|g\|_{L^{\infty}\left(\Gamma^{\perp}\right)} \leq 2\|f\|_{L^{\infty}(G)}
$$

and

$$
\inf _{\gamma \in \mathcal{C}}\left|\widehat{g d \mu_{\Gamma^{\perp}}}(\gamma)\right|>2^{-1} \epsilon^{2} A_{f}^{-1}\|f\|_{L^{\infty}(G)},
$$

so that (9.3) may be rewritten to yield

$$
\sum_{s=0}^{2+\log _{2} \epsilon^{-2} A_{f}} \sum_{\gamma \in \mathcal{C}_{s}}\left|\widehat{g d \mu_{\Gamma^{\perp}}}(\gamma)\right||\widehat{g}(\gamma)| \geq 2^{-1} \epsilon^{2}\|f\|_{L^{\infty}(G)}^{2} .
$$

It follows by the pigeonhole principle that there is some $s$ for which

$$
\sum_{\gamma \in \mathcal{C}_{s}}\left|\widehat{g d \mu_{\Gamma^{\perp}}}(\gamma)\right||\widehat{g}(\gamma)| \gg \frac{\epsilon^{2}\|f\|_{L^{\infty}(G)}^{2}}{1+\log \epsilon^{-1} A_{f}},
$$

and since $\left|\widehat{g d \mu_{\Gamma^{\perp}}}(\gamma)\right| \leq 2^{-(s-1)}\|f\|_{L^{\infty}(G)}$ if $\gamma \in \mathcal{C}_{s}$ we get

$$
\sum_{\gamma \in \mathcal{C}_{s}}|\widehat{g}(\gamma)| \gg \frac{2^{s} \epsilon^{2}\|f\|_{L^{\infty}(G)}}{1+\log \epsilon^{-1} A_{f}}
$$

Now

$$
\mathcal{C}_{s} \subset\left\{\gamma:\left|\widehat{g d \beta^{\prime}}(\gamma)\right|>\left(2^{-s}\|f\|_{L^{\infty}(G)}\|g\|_{L^{\infty}\left(\Gamma^{\perp}\right)}^{-1}\right)\|g\|_{L^{\infty}\left(\Gamma^{\perp}\right)}\right\},
$$

and since $\|g\|_{A\left(\Gamma^{\perp}\right)} \leq\|f\|_{A(G)}$ we may apply Proposition 9.7 to get a set of characters $\Lambda$ such that $\mathcal{C}_{s} \subset(\Gamma \cup \Lambda)^{\perp \perp}$. Moreover $|\Lambda|$ satisfies

$$
\begin{aligned}
|\Lambda| & \ll 2^{s}\|f\|_{L^{\infty}(G)}^{-1}\|g\|_{L^{\infty}\left(\Gamma^{\perp}\right)}\left(1+\log \|g\|_{A\left(\Gamma^{\perp}\right)}\|g\|_{L^{\infty}\left(\Gamma^{\perp}\right)}^{-1}\right) \\
& \ll 2^{s}\|f\|_{L^{\infty}(G)}^{-1}\|g\|_{L^{\infty}\left(\Gamma^{\perp}\right)}^{-1}\left(1+\log \|f\|_{A(G)}\|g\|_{L^{\infty}\left(\Gamma^{\perp}\right)}^{-1}\right) \text { since }\|g\|_{A\left(\Gamma^{\perp}\right)} \leq\|f\|_{A(G)} \\
& \ll 2^{s}\|f\|_{L^{\infty}(G)}^{-1}\|g\|_{L^{\infty}\left(\Gamma^{\perp}\right)}\left(1+\log A_{f}\|f\|_{L^{\infty}(G)}\|g\|_{L^{\infty}\left(\Gamma^{\perp}\right)}^{-1}\right) .
\end{aligned}
$$

So, writing $X$ for $\|f\|_{L^{\infty}(G)}^{-1}\|g\|_{L^{\infty}\left(\Gamma^{\perp}\right)}$ we have

$$
|\Lambda| \ll 2^{s} X\left(1+\log A_{f} X^{-1}\right),
$$

but $\|g\|_{L^{\infty}\left(\Gamma^{\perp}\right)} \leq 2\|f\|_{L^{\infty}(G)}$ so $X \leq 2$ and therefore

$$
|\Lambda| \ll 2^{s} \sup _{X^{\prime} \in(0,2]} X^{\prime}\left(1+\log A_{f} X^{\prime-1}\right) \ll 2^{s}\left(1+\log A_{f}\right) .
$$


The lemma follows.

Iterating this in the same way as before yields Theorem 9.1

\section{The Proof of Theorem 8.4}

We begin by extending the second iteration lemma (Lemma 9.8) from the model setting to that of the general compact Abelian group.

Lemma 10.1. Suppose that $G$ is a compact Abelian group and $B(\Gamma, \delta)$ a regular Bohr set. Suppose that $f \in A(G)$ and $\epsilon \in(0,1]$. Write $A_{f}$ for the quantity $\|f\|_{A(G)}\|f\|_{L^{\infty}(G)}^{-1}$. Then at least one of the following is true.

(1) ( $f$ is close to a continuous function) There is a Bohr set $B\left(\Gamma, \delta^{\prime}\right)$ with $\delta^{\prime} \gg \epsilon \delta / d$ such that

$$
\sup _{x \in G}\|f * \beta-f * \beta(x)\|_{L^{\infty}\left(x+B\left(\Gamma, \delta^{\prime}\right)\right)} \leq \epsilon\|f\|_{L^{\infty}(G)}
$$

and

$$
\sup _{x \in G}\|f-f * \beta\|_{L^{2}\left(x+B\left(\Gamma, \delta^{\prime}\right)\right)} \leq \epsilon\|f\|_{L^{\infty}(G)} .
$$

(2) For all $\eta \in(0,1]$ there is a set of characters $\Lambda, a \delta^{\prime \prime} \in(0,1]$ and a nonnegative integer $s$ with

$$
|\Lambda| \ll 2^{s}\left(1+\log A_{f}\right) \text { and } \delta^{\prime \prime} \gg \epsilon^{5} A_{f}^{-4} \eta \delta / d^{3},
$$

such that

$$
\sum_{\gamma \in \mathcal{L}}|1-\widehat{\beta}(\gamma)||\widehat{f}(\gamma)| \gg \frac{2^{s} \epsilon^{2}\|f\|_{L^{\infty}(G)}}{\min \left\{2^{s}, 1+\log \epsilon^{-1} A_{f}\right\}}
$$

where

$$
\mathcal{L}:=\left\{\gamma:|1-\gamma(x)| \leq \eta \text { for all } x \in B\left(\Gamma \cup \Lambda, \delta^{\prime \prime}\right)\right\} .
$$

Proof. Choosing $\delta^{\prime}$ is easy: By Corollary 6.6 and Proposition 6.3 there is a $\delta^{\prime} \gg \delta \epsilon / d$ regular for $\Gamma$ such that

$$
\sup _{x \in G}\|f * \beta-f * \beta(x)\|_{L^{\infty}\left(x+B\left(\Gamma, \delta^{\prime}\right)\right)} \leq \epsilon\|f\|_{L^{\infty}(G)} .
$$

Now, suppose that

$$
\sup _{x \in G}\|f-f * \beta\|_{L^{2}\left(x+B\left(\Gamma, \delta^{\prime}\right)\right)}>\epsilon\|f\|_{L^{\infty}(G)} .
$$

It follows that there is some $x^{\prime} \in G$ which, without loss of generality, is equal to $0_{G}$ such that

$$
\|f-f * \beta\|_{L^{2}\left(x^{\prime}+B\left(\Gamma, \delta^{\prime}\right)\right)} \geq \epsilon\|f\|_{L^{\infty}(G)} .
$$

For ease of notation write $g=f-f * \beta$, and observe that $g$ satisfies the inequalities

$$
\|g\|_{A(G)} \leq 2\|f\|_{A(G)} \text { and }\|g\|_{L^{\infty}\left(B\left(\Gamma, \delta^{\prime}\right)\right)} \leq 2\|f\|_{L^{\infty}(G)} .
$$

To see the first of these note that

$$
\|g\|_{A(G)}=\sum_{\gamma \in \widehat{G}}|1-\widehat{\beta}(\gamma)||\widehat{f}(\gamma)| \leq \sup _{\gamma \in \widehat{G}}|1-\widehat{\beta}(\gamma)|\|f\|_{A(G)} \leq 2\|f\|_{A(G)},
$$

and for the second

$$
\|g\|_{L^{\infty}\left(B\left(\Gamma, \delta^{\prime}\right)\right)} \leq\|g\|_{L^{\infty}(G)} \leq\|f\|_{L^{\infty}(G)}+\|f * \beta\|_{L^{\infty}(G)} \leq 2\|f\|_{L^{\infty}(G)} .
$$


Returning to (10.1) we may apply Plancherel's theorem and then the triangle inequality to give us a Fourier statement:

$$
\sum_{\gamma \in \widehat{G}}\left|\widehat{g d \beta^{\prime}}(\gamma)\left\|\widehat{g}(\gamma) \mid \geq \epsilon^{2}\right\| f \|_{L^{\infty}(G)}^{2} .\right.
$$

The characters supporting large values of $\widehat{g d \beta^{\prime}}$ make the principal contribution to this sum. Specifically put

$$
\mathcal{C}:=\left\{\gamma \in \widehat{G}:\left|\widehat{g d \beta^{\prime}}(\gamma)\right|>\epsilon^{\prime}\|g\|_{L^{\infty}\left(B\left(\Gamma, \delta^{\prime}\right)\right)}\right\},
$$

where

$$
\epsilon^{\prime}:=2^{-2} \epsilon^{2} A_{f}^{-1}\|f\|_{L^{\infty}(G)}\|g\|_{L^{\infty}\left(B\left(\Gamma, \delta^{\prime}\right)\right)}^{-1} .
$$

Then

$$
\begin{aligned}
\sum_{\gamma \notin \mathcal{C}}\left|\widehat{g d \beta^{\prime}}(\gamma) \| \widehat{g}(\gamma)\right| & \leq 2^{-2} \epsilon^{2} A_{f}^{-1}\|f\|_{L^{\infty}(G)} \sum_{\gamma \notin \mathcal{C}}|\widehat{g}(\gamma)| \\
& \leq 2^{-2} \epsilon^{2} A_{f}^{-1}\|f\|_{L^{\infty}(G)}\|g\|_{A(G)} \\
& \leq 2^{-1} \epsilon^{2}\|f\|_{L^{\infty}(G)}^{2} \text { since }\|g\|_{A(G)} \leq 2\|f\|_{A(G)} .
\end{aligned}
$$

Substituting this into (10.2) we conclude that

$$
\sum_{\gamma \in \mathcal{C}}\left|\widehat{g d \beta^{\prime}}(\gamma)\left\|\widehat{g}(\gamma) \mid \geq 2^{-1} \epsilon^{2}\right\| f \|_{L^{\infty}(G)}^{2} .\right.
$$

Write $I_{s}:=\left(2^{-s}\|f\|_{L^{\infty}(G)}, 2^{-(s-1)}\|f\|_{L^{\infty}(G)}\right]$ and partition $\mathcal{C}$ into the sets

$$
\mathcal{C}_{s}:=\left\{\gamma \in \mathcal{C}:\left|\widehat{g d \beta^{\prime}}(\gamma)\right| \in I_{s}\right\} \text { for } 0 \leq s \leq 3+\log _{2} \epsilon^{-2} A_{f} .
$$

Notice that $\left\{\mathcal{C}_{s}: 0 \leq s \leq 3+\log _{2} \epsilon^{-2} A_{f}\right\}$ covers $\mathcal{C}$ since

$$
\sup _{\gamma \in \mathcal{C}}\left|\widehat{g d \beta^{\prime}}(\gamma)\right| \leq \sup _{\gamma \in \widehat{G}}\left|\widehat{g d \beta^{\prime}}(\gamma)\right| \leq\|g\|_{L^{\infty}\left(B\left(\Gamma, \delta^{\prime}\right)\right)} \leq 2\|f\|_{L^{\infty}(G)}
$$

and

$$
\inf _{\gamma \in \mathcal{C}}\left|\widehat{g d \beta^{\prime}}(\gamma)\right|>2^{-2} \epsilon^{2} A_{f}^{-1}\|f\|_{L^{\infty}(G)}
$$

so that (10.3) may be rewritten to yield

$$
\sum_{s=0}^{3+\log _{2} \epsilon^{-2} A_{f}} \sum_{\gamma \in \mathcal{C}_{s}}\left|\widehat{g d \beta^{\prime}}(\gamma)\left\|\widehat{g}(\gamma) \mid \geq 2^{-1} \epsilon^{2}\right\| f \|_{L^{\infty}(G)}^{2} .\right.
$$

Writing $S^{\prime}:=\left\{s \in \mathbb{N}_{0}: 2^{s} \leq 3+\log _{2} \epsilon^{-2} A_{f}\right\}$ and $S^{\prime \prime}:=\left\{s \in \mathbb{N}_{0}: 2^{s}>3+\right.$ $\left.\log _{2} \epsilon^{-2} A_{f}\right\}$ it follows that either

$$
\sum_{s \in S^{\prime}} 2^{-s} \cdot 2^{s} \sum_{\gamma \in \mathcal{C}_{s}}\left|\widehat{g d \beta^{\prime}}(\gamma)\left\|\widehat{g}(\gamma) \mid \geq 2^{-2} \epsilon^{2}\right\| f \|_{L^{\infty}(G)}^{2}\right.
$$

or

$$
\sum_{s \in S^{\prime \prime}} \sum_{\gamma \in \mathcal{C}_{s}}\left|\widehat{g d \beta^{\prime}}(\gamma)\right||\widehat{g}(\gamma)| \geq 2^{-2} \epsilon^{2}\|f\|_{L^{\infty}(G)}^{2} .
$$

By the pigeonhole principle that there is some $s$ for which

$$
\sum_{\gamma \in \mathcal{C}_{s}}\left|\widehat{g d \beta^{\prime}}(\gamma)\right||\widehat{g}(\gamma)| \gg \frac{\epsilon^{2}\|f\|_{L^{\infty}(G)}^{2}}{1+\log \epsilon^{-1} A_{f}} \text { if } 2^{s}>3+\log _{2} \epsilon^{-2} A_{f}
$$


and

$$
\sum_{\gamma \in \mathcal{C}_{s}}\left|\widehat{g d \beta^{\prime}}(\gamma)\right||\widehat{g}(\gamma)| \gg \frac{\epsilon^{2}\|f\|_{L^{\infty}(G)}^{2}}{2^{s}} \text { if } 2^{s} \leq 3+\log _{2} \epsilon^{-2} A_{f} .
$$

i.e. there is some $s$ such that

$$
\sum_{\gamma \in \mathcal{C}_{s}}\left|\widehat{g d \beta^{\prime}}(\gamma)\right||\widehat{g}(\gamma)| \gg \frac{\epsilon^{2}\|f\|_{L^{\infty}(G)}^{2}}{\min \left\{2^{s}, 1+\log \epsilon^{-1} A_{f}\right\}} .
$$

Since $\left|\widehat{g d \beta^{\prime}}(\gamma)\right| \leq 2^{-(s-1)}\|f\|_{L^{\infty}(G)}$ if $\gamma \in \mathcal{C}_{s}$ we get

$$
\sum_{\gamma \in \mathcal{C}_{s}}|\widehat{g}(\gamma)| \gg \frac{2^{s} \epsilon^{2}\|f\|_{L^{\infty}(G)}}{\min \left\{2^{s}, 1+\log \epsilon^{-1} A_{f}\right\}} .
$$

Now

$$
\mathcal{C}_{s} \subset\left\{\gamma:\left|\widehat{g d \beta^{\prime}}(\gamma)\right| \geq\left(2^{-s}\|f\|_{L^{\infty}(G)}\|g\|_{L^{\infty}\left(B\left(\Gamma, \delta^{\prime}\right)\right)}^{-1}\right)\|g\|_{L^{\infty}\left(B\left(\Gamma, \delta^{\prime}\right)\right)}\right\},
$$

and $g \in A(G)$ so we may apply Proposition 7.4 to get a set of characters $\Lambda$ and a $\delta^{\prime \prime}$ regular for $\Gamma \cup \Lambda$ such that

$$
\mathcal{C}_{s} \subset\left\{\gamma:|1-\gamma(x)| \leq \eta \text { for all } x \in B\left(\Gamma \cup \Lambda, \delta^{\prime \prime}\right)\right\} .
$$

Moreover $|\Lambda|$ satisfies

$$
\begin{aligned}
|\Lambda| & \ll 2^{s}\|f\|_{L^{\infty}(G)}^{-1}\|g\|_{L^{\infty}\left(B\left(\Gamma, \delta^{\prime}\right)\right)}\left(1+\log \|g\|_{A(G)}\|g\|_{L^{\infty}\left(B\left(\Gamma, \delta^{\prime}\right)\right)}^{-1}\right) \\
& \ll 2^{s}\|f\|_{L^{\infty}(G)}^{-1}\|g\|_{L^{\infty}\left(B\left(\Gamma, \delta^{\prime}\right)\right)}\left(1+\log 2\|f\|_{A(G)}\|g\|_{L^{\infty}\left(B\left(\Gamma, \delta^{\prime}\right)\right)}^{-1}\right)
\end{aligned}
$$

since $\|g\|_{A(G)} \leq 2\|f\|_{A(G)}$, so

$$
|\Lambda| \ll 2^{s}\|f\|_{L^{\infty}(G)}^{-1}\|g\|_{L^{\infty}\left(B\left(\Gamma, \delta^{\prime}\right)\right)}\left(1+\log 2 A_{f}\|f\|_{L^{\infty}(G)}\|g\|_{L^{\infty}\left(B\left(\Gamma, \delta^{\prime}\right)\right)}^{-1}\right) .
$$

So, writing $X$ for $\|f\|_{L^{\infty}(G)}^{-1}\|g\|_{L^{\infty}\left(B\left(\Gamma, \delta^{\prime}\right)\right)}$ we have

$$
|\Lambda| \ll 2^{s} X\left(1+\log 2 A_{f} X^{-1}\right),
$$

but $\|g\|_{L^{\infty}\left(B\left(\Gamma, \delta^{\prime}\right)\right)} \leq 2\|f\|_{L^{\infty}(G)}$ so $X \leq 2$ and therefore

$$
|\Lambda| \ll 2^{s} \sup _{X^{\prime} \in(0,2]} X^{\prime}\left(1+\log 2 A_{f} X^{\prime-1}\right) \ll 2^{s}\left(1+\log A_{f}\right) .
$$

Furthermore $\delta^{\prime \prime}$ satisfies

$$
\begin{aligned}
\delta^{\prime \prime} & \gg 2^{-2 s}\|f\|_{L^{\infty}(G)}^{2}\|g\|_{L^{\infty}\left(B\left(\Gamma, \delta^{\prime}\right)\right)}^{-2} \eta \delta^{\prime} / d^{2}\left(1+\log \|g\|_{A(G)}\|g\|_{L^{\infty}\left(B\left(\Gamma, \delta^{\prime}\right)\right)}^{-1}\right) \\
& \gg 2^{-2 s}\|f\|_{L^{\infty}(G)}^{2} \eta \delta^{\prime} / d^{2}\|g\|_{A(G)}^{2} \\
& \gg \epsilon^{4} A_{f}^{-2}\|f\|_{L^{\infty}(G)}^{2} \eta \delta^{\prime} / d^{2}\|g\|_{A(G)}^{2} \text { since } 2^{2 s} \leq 2^{4} \epsilon^{-4} A_{f}^{2} \\
& \gg \epsilon^{4} A_{f}^{-4} \eta \delta^{\prime} / d^{2} \text { since }\|g\|_{A(G)} \leq 2\|f\|_{A(G)} .
\end{aligned}
$$

The lemma follows.

We are now in a position to iterate this lemma.

Proof of Theorem 8.4. Fix $\eta$ to be optimized at the end of the argument. We construct a sequence of regular Bohr sets $B\left(\Gamma_{k}, \delta_{k}\right)$ iteratively using Lemma 10.1 Put

$$
\mathcal{L}_{k}:=\left\{\gamma:|1-\gamma(x)| \leq \eta \text { for all } x \in B\left(\Gamma_{k}, \delta_{k}\right)\right\}
$$


and

$$
d_{k}:=\left|\Gamma_{k}\right| \text { and } L_{k}:=\sum_{\gamma \in \mathcal{L}_{k}}|\widehat{f}(\gamma)|
$$

We initialize the iteration with $\Gamma_{0}:=\left\{0_{\widehat{G}}\right\}$ and $\delta_{0} \gg 1$ regular for $\Gamma_{0}$, chosen so by Proposition 6.3

Suppose that we are at stage $k$. Apply the iteration lemma (Lemma 10.1) to $f$ and the regular Bohr set $B\left(\Gamma_{k}, \delta_{k}\right)$. If we are in the first case terminate with the desired conclusion; if not then we get a set of characters $\Lambda$, a $\delta^{\prime \prime} \in(0,1]$ and an integer $s$. Let $\Gamma_{k+1}=\Gamma_{k} \cup \Lambda$, pick $\delta_{k+1} \in\left(\delta^{\prime \prime} / 2, \delta^{\prime \prime}\right]$ regular for $\Gamma_{k+1}$ by Proposition 6.3. and let $s_{k+1}=s$. We are given that

$$
d_{k+1}-d_{k} \ll 2^{s_{k+1}}\left(1+\log A_{f}\right) \text { and } \delta_{k+1} \gg \epsilon^{5} A_{f}^{-4} \eta \delta_{k} / d_{k}^{3},
$$

and furthermore

$$
2\left(L_{k+1}-L_{k}\right)+\eta L_{k} \gg \frac{2^{s_{k+1}} \epsilon^{2}\|f\|_{L^{\infty}(G)}}{\min \left\{2^{s_{k+1}}, 1+\log \epsilon^{-1} A_{f}\right\}} .
$$

Since $L_{k} \leq\|f\|_{A(G)}$ and $s_{k} \geq 0$ it follows that we can pick $\eta \gg \epsilon^{3} A_{f}^{-2}$ (independently of $k$ ) such that

$$
L_{k+1}-L_{k} \gg \frac{2^{s_{k+1}} \epsilon^{2}\|f\|_{L^{\infty}(G)}}{\min \left\{2^{s_{k+1}}, 1+\log \epsilon^{-1} A_{f}\right\}} .
$$

Hence by induction we have

$$
L_{k} \gg \epsilon^{2}\|f\|_{L^{\infty}(G)} \sum_{l=1}^{k} \frac{2^{s_{l}}}{\min \left\{2^{s_{l}}, 1+\log \epsilon^{-1} A_{f}\right\}} \text { and } d_{k} \ll \sum_{l=1}^{k} 2^{s_{l}}\left(1+\log A_{f}\right) .
$$

Again since $s_{k} \geq 0$ it follows that the iteration terminates. Hence we have

$$
\sum_{l=1}^{k} 2^{s_{l}} \ll L_{k} \epsilon^{-2}\|f\|_{L^{\infty}(G)}^{-1}\left(1+\log \epsilon^{-1} A_{f}\right) \ll \epsilon^{-2} A_{f}\left(1+\log \epsilon^{-1} A_{f}\right),
$$

since $\|f\|_{A(G)} \geq L_{k}$. It follows that

$$
d_{k} \ll \epsilon^{-2} A_{f}\left(1+\log A_{f}\right)\left(1+\log \epsilon^{-1} A_{f}\right) .
$$

The bound on $\eta$ and $d_{k}$ gives us

$$
\delta_{k+1} \gg \epsilon^{17} A_{f}^{-15} \delta_{k},
$$

and hence

$$
\begin{aligned}
\log \delta_{k}^{-1} & \ll k\left(1+\log \epsilon^{-1} A_{f}\right) \\
& \ll \sum_{l=1}^{k} \frac{2^{s_{k}}}{\min \left\{2^{s_{k}}, 1+\log \epsilon^{-1} A_{f}\right\}}\left(1+\log \epsilon^{-1} A_{f}\right) \\
& \ll \epsilon^{-2} A_{f}\left(1+\log \epsilon^{-1} A_{f}\right) .
\end{aligned}
$$

The result follows. 


\section{The proof of Theorem 1.4 And CONCLUding Remarks}

Having proved Theorem 8.4 it is essentially a formality to carry out the rest of the argument detailed in $₫ 8$.

Proof of Theorem 1.4. Write $G$ for $\mathbb{Z} / p \mathbb{Z}$ and $\alpha:=\mu_{G}(A)=|A| / p$. We apply Theorem 8.4 to $f=\chi_{A}$ with $\epsilon=2^{-2} \alpha(1-\alpha)$. This gives a Bohr set $B(\Gamma, \delta)$ with

$$
d \ll_{\alpha}\left\|\chi_{A}\right\|_{A(G)}\left(1+\log \left\|\chi_{A}\right\|_{A(G)}\right)^{2}
$$

and

$$
\log \delta^{-1} \ll_{\alpha}\left\|\chi_{A}\right\|_{A(G)}\left(1+\log \left\|\chi_{A}\right\|_{A(G)}\right),
$$

and a narrower Bohr set $B\left(\Gamma, \delta^{\prime}\right)$ with $\delta^{\prime} \gg_{\alpha} \delta / d$ such that

$$
\sup _{x \in G}\left\|\chi_{A} * \beta-\chi_{A} * \beta(x)\right\|_{L^{\infty}\left(x+B\left(\Gamma, \delta^{\prime}\right)\right)} \leq 2^{-2} \alpha(1-\alpha)
$$

and

$$
\sup _{x \in G}\left\|\chi_{A}-\chi_{A} * \beta\right\|_{L^{2}\left(x+B\left(\Gamma, \delta^{\prime}\right)\right)} \leq 2^{-2} \alpha(1-\alpha) .
$$

Suppose that $\mu_{G}\left(B\left(\Gamma, \delta^{\prime}\right)\right)>p^{-1}$. Then there is a non-zero $y \in B\left(\Gamma, \delta^{\prime}\right)$, and such a $y$ has the property that $\left|\chi_{A} * \beta(x+y)-\chi_{A} * \beta(x)\right| \leq 2^{-2} \alpha(1-\alpha)$ for all $x \in G$. It follows that we may apply the discrete intermediate value theorem (Proposition 8.2) to $\chi_{A} * \beta$ and conclude that there is some $x \in G$ such that

$$
\left|\chi_{A} * \beta(x)-\alpha\right| \leq 2^{-3} \alpha(1-\alpha) .
$$

Furthermore (11.1) ensures that there is some $x^{\prime} \in x+B\left(\Gamma, \delta^{\prime}\right)$ such that

$$
\left|\chi_{A}\left(x^{\prime}\right)-\chi_{A} * \beta\left(x^{\prime}\right)\right| \leq 2^{-2} \alpha(1-\alpha),
$$

and this gives

$$
\begin{aligned}
\left|\chi_{A}\left(x^{\prime}\right)-\alpha\right| \leq & \left|\chi_{A}\left(x^{\prime}\right)-\chi_{A} * \beta\left(x^{\prime}\right)\right| \\
& +\left|\chi_{A} * \beta\left(x^{\prime}\right)-\chi_{A} * \beta(x)\right| \\
& +\left|\chi_{A} * \beta(x)-\alpha\right| \\
\leq & \alpha(1-\alpha) .
\end{aligned}
$$

This contradicts the fact that $\chi_{A}\left(x^{\prime}\right) \in\{0,1\}$, and hence $\mu_{G}\left(B\left(\Gamma, \delta^{\prime}\right)\right) \leq p^{-1}$. Lemma 6.2 then lets us infer that $d\left(1+\log \delta^{\prime-1}\right) \gg \log p$ from which, on inserting the bounds on $d$ and $\delta^{\prime}$, the result follows.

In GK09 Green and Konyagin essentially prove a version of Theorem 8.4 with different bounds.

Theorem 11.1. Suppose that $G$ is a compact Abelian group, $f \in A(G)$ and $\epsilon \in$ $(0,1]$. Write $A_{f}$ for the quantity $\|f\|_{A(G)}\|f\|_{\infty}^{-1}$. Then there is a Bohr set $B(\Gamma, \delta)$ with

$$
|\Gamma| \ll \epsilon^{-2} A_{f}^{2} \text { and } \log \delta^{-1} \ll \epsilon^{-1} A_{f}\left(1+\log \epsilon^{-1} A_{f}\right),
$$

and a narrower Bohr set $B\left(\Gamma, \delta^{\prime}\right)$ with $\delta^{\prime} \gg \epsilon \delta / d$ such that

$$
\sup _{x \in G}\|f * \beta-f * \beta(x)\|_{L^{\infty}\left(x+B\left(\Gamma, \delta^{\prime}\right)\right)} \leq \epsilon\|f\|_{L^{\infty}(G)}
$$

and

$$
\sup _{x \in G}\|f-f * \beta\|_{L^{2}\left(x+B\left(\Gamma, \delta^{\prime}\right)\right)} \leq \epsilon\|f\|_{L^{\infty}(G)} .
$$


The crucial difference between our proof of Theorem 8.4 and their proof of Theorem 11.1 is that in their iteration lemma they find only a few characters at which $\widehat{f}$ is large, whereas we find all characters at which $\widehat{f}$ is large. Their approach leads to superior bounds in the basic version of their argument, however it prevents them from using a tool such as Proposition 7.4, which is where our argument gains its edge.

In both the arguments of Green and Konyagin and of this paper the width of the Bohr set which one eventually finds narrows exponentially with the number of times one has to use the (appropriate) iteration lemma. Green and Konyagin employ a neat trick to reduce this - the natural version of their argument has $\log \delta^{-1} \ll \epsilon^{-2} A_{f}^{2}\left(1+\log \epsilon^{-1} A_{f}\right)$ - which leads to the superior $\epsilon$-dependence for $\log \delta^{-1}$ in Theorem 11.1. It is possible to add their trick to our argument and hence improve the $\epsilon$-dependence of $\log \delta^{-1}$ in Theorem 8.4 too, however this would have no effect on our application.

The model setting of $\mathbb{F}_{2}^{n}$, has been used extensively in this paper to make our results clearer; the paper Gre05] of Green serves as a good survey of other problems where it has found similar uses. While the primary rôle of the model setting is one of clarification, the main question of this paper can nevertheless be tackled in $\mathbb{F}_{2}^{n}$, and in particular the following, for example, is proved in San07.

Theorem 11.2. Suppose that $G=\mathbb{F}_{2}^{n}$ and $A \subset G$ has density $\alpha$ with $|\alpha-1 / 3| \leq \epsilon$. Then

$$
\left\|\chi_{A}\right\|_{A(G)} \gg \log \log \epsilon^{-1} .
$$

Finally it would be interesting to know what the true bounds in Theorem 8.4 should be. As far as the model analogue, Theorem 9.1, is concerned it would probably be surprising if one could beat the following.

Conjecture 11.3. Suppose that $G=\mathbb{F}_{2}^{n}, f \in A(G)$ and $\epsilon \in(0,1]$. Write $A_{f}$ for the quantity $\|f\|_{A(G)}\|f\|_{L^{\infty}(G)}^{-1}$. Then there is a subspace $V$ of $G$ with

$$
\operatorname{codim} V \ll \epsilon^{-2} A_{f}
$$

and

$$
\sup _{x^{\prime} \in G}\left\|f-f\left(x^{\prime}\right)\right\|_{L^{2}\left(x^{\prime}+V\right)} \leq \epsilon\|f\|_{L^{\infty}(G)} .
$$

It is, however, not clear what an argument giving this might provide in the general setting. If the argument is iterative in the style of this paper then to provide an improvement in the exponent of $\log p$ in Theorem 1.4 one would require some way of cutting down the number of times we iterate.

\section{ACKNOWLEDGMENTS}

I should like to thank Tim Gowers for reviewing many drafts of the paper, Ben Green for guidance and innumerable valuable conversations, Ben Green and Sergei Konyagin for making some early drafts of the preprint GK09 available, and an anonymous referee for a number of useful suggestions and improvements.

\section{Appendix A. The general COnstruction of the AuXiliary measure}

We extend the approach of 4 from the model setting to that of $G$ an arbitrary compact Abelian group. Here Riesz products are marginally more complicated. 
A.1. Riesz products. Suppose that $\Lambda$ is a finite set of characters. We say that $\omega \in \ell^{\infty}\left(\Lambda \cup \Lambda^{-1}\right)$ is hermitian if

$$
\omega\left(\lambda^{-1}\right)=\overline{\omega(\lambda)} \text { for all } \lambda \in \Lambda ;
$$

if $\omega$ also satisfies $\|\omega\|_{\infty} \leq 1$ then we define the product

$$
p_{\omega}:=\prod_{\lambda \in \Lambda}\left(1+\frac{\omega(\lambda) \lambda+\omega\left(\lambda^{-1}\right) \lambda^{-1}}{2}\right)
$$

Such a product is called a Riesz product and it is easy to see that it is real and non-negative from which it follows that $\left\|p_{\omega}\right\|_{1}=\widehat{p_{\omega}}\left(0_{\widehat{G}}\right)$. Further expanding out the product reveals that $\operatorname{supp} \widehat{p_{\omega}} \subset\langle\Lambda\rangle$.

We had an easy time computing the Fourier transform of Riesz products in $\mathbb{F}_{2}^{n}$, in general it is more complicated. We can expand out the product in (A.1) to see that:

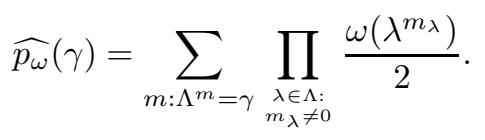

To keep track of this we say that $\widetilde{p}$, defined on $\{-1,0,1\}^{\Lambda}$, is a formal Fourier transform 1 for $p \in L^{1}(G)$ if

$$
\widehat{p}(\gamma)=\sum_{m: \Lambda^{m}=\gamma} \widetilde{p}(m) \text { for all } \gamma \in \widehat{G} .
$$

The functions which we are interested in are of the form

$$
p(x):=\int p_{t \omega}(x) d \tau(t),
$$

for $\omega \in \ell^{\infty}\left(\Lambda \cup \Lambda^{-1}\right)$ hermitian with $\|\omega\|_{\infty} \leq 1$, and $\tau$ a real measure on $[-1,1]$. It follows from (A.2) and linearity of the Fourier transform that $\widetilde{p}$ defined by

$$
\widetilde{p}(m):=\int t^{|m|} d \tau(t) . \prod_{\substack{\lambda \in \Lambda: \\ m_{\lambda} \neq 0}} \frac{\omega\left(\lambda^{m_{\lambda}}\right)}{2} \text { for all } m \in\{-1,0,1\}^{\Lambda},
$$

is a formal Fourier transform for $p$.

If $\Lambda$ is dissociated then when $\gamma=0_{\widehat{G}}$ there is only one summand in the expression for $\widehat{p_{\omega}}(\gamma)$ given in (A.2) and that has a value of 1 , so by non-negativity of $p_{\omega}$

$$
\left\|p_{\omega}\right\|_{1}=\widehat{p_{\omega}}\left(0_{\widehat{G}}\right)=1 .
$$

Dissociativity makes computing the Fourier transform easy for $\gamma=0_{\widehat{G}}$ by restricting the number of non-zero summands in A.2); a lemma of Rider Rid66. provides a result for more general $\gamma$ :

\footnotetext{
${ }^{1}$ Formal Fourier transforms are not in general unique.
} 
Lemma A.2. Suppose that $G$ is a compact Abelian group and $\Lambda$ is a finite dissociated set of characters on $G \sqrt{2}$ Then for all $\gamma \in \widehat{G}$

$$
\left|\left\{m \in\{-1,0,1\}^{\Lambda}:|m|=r, \Lambda^{m}=\gamma\right\}\right| \leq 2^{r} .
$$

Proof. Let $\omega$ be the hermitian function which takes $\Lambda$ to 1 . For this choice of $\omega$ (A.2) is

$$
\widehat{p_{\omega}}(\gamma)=\sum_{r \geq 0} 2^{-r}\left|\left\{m \in\{-1,0,1\}^{\Lambda}:|m|=r, \Lambda^{m}=\gamma\right\}\right|
$$

$\operatorname{But}\left|\widehat{p_{\omega}}(\gamma)\right| \leq\left\|p_{\omega}\right\|_{1}=1$ since $\Lambda$ is dissociated which yields the conclusion.

Proposition A.3. Suppose that $G$ is a compact Abelian group, $\Lambda$ a finite dissociated set of characters on $G$ with no elements of order 2 and $\omega \in \ell^{\infty}\left(\Lambda \cup \Lambda^{-1}\right)$ is hermitian with $\|\omega\|_{\infty} \leq 1$. Then for any $\eta \in(0,1]$ there is a function $f_{\eta} \in L^{1}(G)$ such that

$$
\left.\widehat{f}_{\eta}\right|_{\Lambda \cup \Lambda^{-1}}=\omega,\left\|f_{\eta}\right\|_{1} \ll\left(1+\log \eta^{-1}\right) \text { and }\left|\widehat{f}_{\eta}(\gamma)\right| \leq \eta \text { for all } \gamma \notin \Lambda \cup \Lambda^{-1} .
$$

Proof. Fix an integer $l>1$ to be optimized later and let $\tau_{2 l}$ be the measure of Lemma 4.5. Define

$$
p(x):=\int p_{t \omega} d \tau_{2 l}(t)
$$

and let $\widetilde{p}$ be the formal Fourier transform for $p$ defined by A.4 $) . \widetilde{p}(m)=0$ if $|m|=0$ by definition of $\tau_{2 l}$ and $\widetilde{p}$, so

$$
\begin{aligned}
\left|\widehat{p}(\gamma)-\sum_{\substack{|m|=1 \\
\Lambda^{m}=\gamma}} \widetilde{p}(m)\right| & \leq \sum_{r \geq 2} \sum_{\substack{|m|=r \\
\Lambda^{m}=\gamma}}|\widetilde{p}(m)| \text { by definition (A.3), } \\
& \leq \sum_{\substack { r>2 l \\
\begin{subarray}{c}{|m|=r \\
\Lambda^{m}=\gamma{ r > 2 l \\
\begin{subarray} { c } { | m | = r \\
\Lambda ^ { m } = \gamma } }\end{subarray}}|\widetilde{p}(m)| \text { since } \int t^{r} d \tau_{2 l}(t)=0 \text { for } 1<r \leq 2 l, \\
& \leq \sum_{r>2 l} \sup _{\substack{|m|=r \\
\Lambda^{m}=\gamma}}\left|\widetilde{p}(m) \|\left\{m:|m|=r, \Lambda^{m}=\gamma\right\}\right| \\
& \leq \sum_{r>2 l} 2^{r} \sup _{\substack{|m|=r \\
\Lambda^{m}=\gamma}}|\widetilde{p}(m)| \text { by Lemma A.2. } \\
& \leq \sum_{r>2 l} 2^{r} \int t^{r} d \tau_{2 l}(t)\left(2^{-1}\|\omega\|_{\infty}\right)^{r} \\
& \leq \sum_{r>2 l} 2\left(2^{-1}\|\omega\|_{\infty}\right)^{r} \text { since }\left|\int t^{r} d \tau_{2 l}(t)\right| \leq 2^{1-r}, \\
& \leq 2^{1-2 l}\|\omega\|_{\infty} .
\end{aligned}
$$

Lemma A.2 applies above because $\Lambda$ is dissociated. Now let $l$ be such that $2^{3-2 l} \leq \eta$ but $l \ll\left(1+\log \eta^{-1}\right)$ and put $f_{\eta}^{(1)}:=2 p$. Then

\footnotetext{
${ }^{2}$ The definition of dissociativity did not require any topological structure on the group and indeed Rider's lemma is true without the assumption that $\Lambda$ is a set of characters for simple reasons of duality: if $H$ is the group generated by $\Lambda$ then we can endow it with the discrete topology and embed $\Lambda$ in $\widehat{\widehat{H}}$. The image of $\Lambda$ under this embedding is a dissociated set of characters on the compact Abelian group $\widehat{H}$, so that there is no real loss of generality in assuming that $\Lambda$ is a set of characters.
} 
(1) If $\gamma \in \Lambda \cup \Lambda^{-1}$ then

$$
\sum_{\substack{|m|=1 \\ \Lambda^{m}=\gamma}} \widetilde{p}(m)=\int t d \tau_{2 l}(t) \frac{\omega(\gamma)}{2}=\frac{\omega(\gamma)}{2}
$$

since $\Lambda$ has no elements of order 2 . Hence by A.5

$$
\left|\widehat{f_{\eta}^{(1)}}(\gamma)-\omega(\gamma)\right| \leq 2^{2-2 l}\|\omega\|_{\infty} \leq 2^{-1}\|\omega\|_{\infty} .
$$

(2) If $\gamma \notin \Lambda \cup \Lambda^{-1}$ then

$$
\sum_{\substack{|m|=1 \\ \Lambda m=\gamma}} \widetilde{p}(m)=0
$$

so by (A.5)

$$
\left|\widehat{f_{\eta}^{(1)}}(\gamma)\right| \leq 2^{-1} \eta\|\omega\|_{\infty}
$$

(3) $\left\|f_{\eta}^{(1)}\right\| \leq 2\left\|\tau_{2 l}\right\|$ by the definition of $p$ and the triangle inequality.

(4) $\left.\widehat{f_{\eta}^{(1)}}\right|_{\Lambda \cup \Lambda^{-1}}$ is hermitian since $\tau_{2 l}$ is real.

We apply the foregoing recursively to the hermitian functions $\omega,\left.2\left(\omega-\widehat{f_{\eta}^{(1)}}\right)\right|_{\Lambda \cup \Lambda^{-1}}$, $\left.2\left(2\left(\omega-\widehat{f_{\eta}^{(1)}}\right)-\widehat{f_{\eta}^{(2)}}\right)\right|_{\Lambda \cup \Lambda^{-1}}, \ldots$ to get a sequence of $L^{1}(G)$-functions $f_{\eta}^{(1)}, f_{\eta}^{(2)}, f_{\eta}^{(3)}$ ,... such that:

(1) If $\gamma \in \Lambda \cup \Lambda^{-1}$ then

$$
\left|\sum_{k=1}^{n} 2^{-(k-1)} \widehat{f_{\eta}^{(k)}}(\gamma)-\omega(\gamma)\right| \leq 2^{-n} .
$$

(2) If $\gamma \notin \Lambda \cup \Lambda^{-1}$ then

$$
\left|\sum_{k=1}^{n} 2^{-(k-1)} \widehat{f_{\eta}^{(k)}}(\gamma)\right| \leq \sum_{k=1}^{n} 2^{-(k-1)} \cdot \frac{\eta}{2} \leq \eta .
$$

$$
\left\|\sum_{k=1}^{n} 2^{-(k-1)} f_{\eta}^{(k)}\right\|_{1} \leq \sum_{k=1}^{n} 2^{-(k-1)}\left\|f_{\eta}^{(k)}\right\|_{1} \leq 2^{2}\left\|\tau_{2 l}\right\|
$$

The sum $\sum_{k=1}^{n} 2^{-(k-1)} f_{\eta}^{(k)}$ converges to a function $f_{\eta} \in L^{1}(G)$ with the required properties since $\left\|\tau_{2 l}\right\| \ll l \ll\left(1+\log \eta^{-1}\right)$.

Finally we modify the above proposition so that the Fourier transform is small on $\Lambda^{-1} \backslash \Lambda$.

Proof of Proposition 3.3. Let $G^{\prime}:=G \times S^{1}$ and identify its dual with $\widehat{G} \times \mathbb{Z}$; let $\Lambda^{\prime}=\Lambda \times\{1\}$, which is dissociated since $\Lambda$ is dissociated, and has no elements of order 2 since 1 is not of order 2 in $\mathbb{Z}$; let $\omega^{\prime}$ be the hermitian map on $\Lambda^{\prime} \cup \Lambda^{\prime-1}$ induced by $\omega^{\prime}(\lambda, 1):=\omega(\lambda)$. Apply Proposition A.3 to $G^{\prime}, \Lambda^{\prime}$ and $\omega^{\prime}$ to get the function $f_{\eta} \in L^{1}\left(G^{\prime}\right)$. Let $\mu_{\eta}$ be the measure induced by the functional

$$
f \mapsto \int_{(x, z) \in G^{\prime}} f(x) f_{\eta}(x, z) \bar{z} d \mu_{G^{\prime}}(x, z)
$$


on $C(G)$. If $\gamma \in \widehat{G}$ then

$$
\widehat{\mu_{\eta}}(\gamma)=\int_{(x, z) \in G^{\prime}} \bar{\gamma}(x) f_{\eta}(x, z) \bar{z} d \mu_{G^{\prime}}(x, z)=\widehat{f_{\eta}}(\gamma, 1) .
$$

We verify the three properties of $\mu_{\eta}$ from the corresponding properties of $f_{\eta}$ :

(1) If $\lambda \in \Lambda$ then $\widehat{\mu_{\eta}}(\lambda)=\widehat{f_{\eta}}(\lambda, 1)=\omega^{\prime}(\lambda, 1)=\omega(\lambda)$.

(2)

$$
\begin{aligned}
\left\|\mu_{\eta}\right\| & =\sup _{f \in C(G):\|f\|_{\infty} \leq 1}\left|\int_{(x, z) \in G^{\prime}} \bar{\gamma}(x) f_{\eta}(x, z) \bar{z} d \mu_{G^{\prime}}(x, z)\right| \\
& \leq \int_{(x, z) \in G^{\prime}}\left|f_{\eta}(x, z)\right| d \mu_{G^{\prime}} \\
& \ll\left(1+\log \eta^{-1}\right) .
\end{aligned}
$$

(3) If $\gamma \notin \Lambda$ then $(\gamma, 1) \notin \Lambda^{\prime} \cup \Lambda^{\prime-1}$ so $\left|\widehat{\mu_{\eta}}(\gamma)\right| \leq \eta$.

\section{REFERENCES}

[Bou99] J. Bourgain. On triples in arithmetic progression. Geom. Funct. Anal., 9(5):968-984, 1999.

[Cha00] B Chazelle. The discrepancy method. Cambridge University Press, Cambridge, 2000. Randomness and complexity.

[Cha02] M.-C. Chang. A polynomial bound in Freŭman's theorem. Duke Math. J., 113(3):399-419, 2002.

[Dru72] S. W. Drury. Unions of sets of interpolation. In Conference on Harmonic Analysis (Univ. Maryland, College Park, Md., 1971), pages 23-33. Lecture Notes in Math., Vol. 266. Springer, Berlin, 1972.

[GK09] B. J. Green and S. V. Konyagin. On the Littlewood problem modulo a prime. Canad. J. Math., 61(1):141-164, 2009.

[Gre04] B. J. Green. Spectral structure of sets of integers. In Fourier analysis and convexity, Appl. Numer. Harmon. Anal., pages 83-96. Birkhäuser Boston, Boston, MA, 2004.

[Gre05] B. J. Green. Finite field models in additive combinatorics. In Surveys in combinatorics 2005, volume 327 of London Math. Soc. Lecture Note Ser., pages 1-27. Cambridge Univ. Press, Cambridge, 2005.

[GT08] B. J. Green and T. C. Tao. An inverse theorem for the Gowers $U^{3}(G)$ norm. Proc. Edinb. Math. Soc. (2), 51(1):73-153, 2008.

[Hal81] G. Halász. On Roth's method in the theory of irregularities of point distributions. In Recent progress in analytic number theory, Vol. 2 (Durham, 1979), pages 79-94. Academic Press, London, 1981.

[Mél82] J.-F. Méla. Mesures $\varepsilon$-idempotentes de norme bornée. Studia Math., 72(2):131-149, 1982.

[Rid66] D. Rider. Gap series on groups and spheres. Canad. J. Math., 18:389-398, 1966.

[Rud90] W. Rudin. Fourier analysis on groups. Wiley Classics Library. John Wiley \& Sons Inc., New York, 1990. Reprint of the 1962 original, A Wiley-Interscience Publication.

[San07] T. Sanders. The $\ell^{1}$-norm of the Fourier transform on compact vector spaces. Bull. Lond. Math. Soc., 39(3):509-521, 2007.

[San08] T. Sanders. Additive structures in sumsets. Math. Proc. Cambridge Philos. Soc., 144(2):289-316, 2008.

Department of Pure Mathematics and Mathematical Statistics, Centre for Mathematical Sciences, Wilberforce Road, Cambridge CB3 0WA, England

E-mail address: t.sanders@dpmms.cam.ac.uk 